\title{
Analysis of elastic incoherent neutron scattering data beyond the Gaussian approximation
}

Cite as: J. Chem. Phys. 149, 234908 (2018); https://doi.org/10.1063/1.5049938

Submitted: 26 July 2018 . Accepted: 27 November 2018 . Published Online: 21 December 2018

D. Zeller, M. T. F. Telling, M. Zamponi, V. García Sakai, and J. Peters (i)

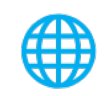

\section{ARTICLES YOU MAY BE INTERESTED IN}

Perspective: Searching for simplicity rather than universality in glass-forming liquids The Journal of Chemical Physics 149, 230901 (2018); https://doi.org/10.1063/1.5048093

A semianalytical "reverse" approach to link structure and microscopic interactions in twoYukawa competing fluids

The Journal of Chemical Physics 149, 234907 (2018); https://doi.org/10.1063/1.5047448

Communication: Relating the pure and ensemble density matrix functional

The Journal of Chemical Physics 149, 231102 (2018); https://doi.org/10.1063/1.5080088

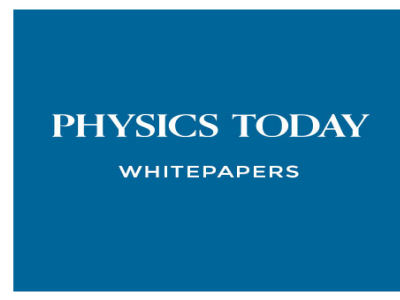

ADVANCED LIGHT CURE ADHESIVES

Take a closer look at what these

environmentally friendly adhesive systems can do

\section{READ NOW}

PRESENTED BY 8 MASTERBOND" 


\title{
Analysis of elastic incoherent neutron scattering data beyond the Gaussian approximation
}

\author{
D. Zeller,,${ }^{1,2,3}$ M. T. F. Telling, ${ }^{3}$ M. Zamponi, ${ }^{4}$ V. García Sakai, ${ }^{3}$ and J. Peters ${ }^{1,2, a)}$ \\ ${ }^{1}$ Université Grenoble Alpes, CNRS, LiPhy, 140 av. de la Physique, 38000 Grenoble, France \\ ${ }^{2}$ Institut Laue-Langevin, 71 Avenue des Martyrs, 38042 Grenoble Cedex 9, France \\ ${ }^{3}$ ISIS Pulsed Neutron and Muon Source, STFC Rutherford Appleton Laboratory, Chilton, \\ Oxfordshire OX11 OQX, United Kingdom \\ ${ }^{4}$ Forschungszentrum Jülich GmbH, Jülich Centre for Neutron Science (JCNS) at Heinz Maier-Leibnitz, Zentrum \\ (MLZ), Lichtenbergstr. 1, 85748 Garching, Germany
}

(Received 26 July 2018; accepted 27 November 2018; published online 21 December 2018)

\begin{abstract}
This work addresses the use of the Gaussian approximation as a common tool to extract atomic motions in proteins from elastic incoherent neutron scattering and whether improvements in data analysis and additional information can be obtained when going beyond that. We measured alphalactalbumin with different levels of hydration on three neutron backscattering spectrometers, to be able to resolve a wide temporal and spatial range for dynamics. We demonstrate that the Gaussian approximation gives qualitatively similar results to models that include heterogeneity, if one respects a certain procedure to treat the intercept of the elastic intensities with the momentum transfer axis. However, the inclusion of motional heterogeneity provides better fits to the data. Our analysis suggests an approach of limited heterogeneity, where including only two kinds of motions appears sufficient to obtain more quantitative results for the mean square displacement. Finally, we note that traditional backscattering spectrometers pose a limit on the lowest accessible momentum transfer. We therefore suggest that complementary information about the spatial evolution of the elastic intensity close to zero momentum transfer can be obtained using other neutron methods, in particular, neutron spin-echo together with polarization analysis. Published by AIP Publishing. https://doi.org/10.1063/1.5049938
\end{abstract}

\section{INTRODUCTION}

Neutron scattering techniques have been successfully employed to study biological systems for about 50 years. ${ }^{1-3}$ Neutron scattering from any sample gives rise to two contributions, a coherent and an incoherent part. ${ }^{4}$ Whereas coherent scattering requires a constant phase relation to be maintained between the incident and scattered neutron, giving access to structural or collective motional information, incoherent scattering is a probe of the average motions of individual particles within the sample and thus molecular dynamics. ${ }^{4}$ In the present work, we concentrate on the incoherent part assuming that most of the signal comes from the dominant incoherent scattering from the sizable number of protons that are distributed throughout the sample. The incoherent scattering signal can be further subdivided into an elastic part, corresponding to those atoms in the sample that move with a characteristic time scale that falls outside the temporal resolution of the neutron instrument used and thus are not seen to exchange energy with the neutron upon scattering, called the Elastic Incoherent Neutron Scattering (EINS), and those whose motions lead to an energy exchange with the neutron. Traditionally, one distinguishes here quasi-elastic neutron scattering (QENS), where only small amounts of energy are exchanged (typically $<2 \mathrm{~m} \mathrm{eV}$ ) and which manifests itself as a broadening of the zero-energy

a)Electronic mail: jpeters@ill.fr transfer centered elastic peak, from inelastic neutron scattering (INS), which appears as satellite peaks well separated from the elastic part and correspond to specific modes or excitations within the sample. ${ }^{4}$ For the analysis of QENS data, the approach suggested by Van Hove ${ }^{5}$ based on pair correlation functions is most commonly applied and is nearly the exclusive model used so far. Only recently, alternative approaches were suggested by Frauenfelder et al. ${ }^{6}$ and Kneller. ${ }^{7}$ However, new formalisms have to be tested and validated against experimental data, which is done in the present study for EINS.

EINS is generally used to compare the dynamical behavior of biomolecules, especially proteins. ${ }^{3,8,9}$ For this, mean square displacements (MSDs) of the atoms in the sample are extracted over a wide temperature range. The MSDs are considered as a measure of the sample flexibility at a given temperature. ${ }^{10}$ Most biomolecules undergo transitions, which are also visible in the MSD by a change of the slope. For instance, proteins show a dynamical transition at around $220 \mathrm{~K}$ which characterizes the crossover from harmonic to anharmonic motions. ${ }^{2}$ Zaccai introduced an empirical parameter, known as the effective force constant or resilience, ${ }^{11}$ and it corresponds to the slope of the MSD before and after the dynamical transition, which is used to quantify the protein's stability in a given state. Similarly, lipids undergo structural phase transitions which can also be detected by a variation in the dynamics through a change in the MSD. ${ }^{12}$ Thus, EINS is a standard mode of measurement on neutron backscattering 
spectrometers. Despite such a common use, scientists often compare the MSD obtained from a set of samples measured under the same conditions and on the same instrument, only. This is because the obtained absolute values of the MSDs are not always suited for a quantitative comparison with results from other techniques. Moreover, if one searches for specific motions whose amplitudes are known, as for instance to differentiate 2 - or 3 -site jump motions, ${ }^{13}$ precise spatial information would be highly desirable. The aim of the present study is thus to improve the data evaluation and to obtain more certainty about the quantitative accuracy of MSD analysis and results. Such results could, in addition, be used to validate molecular dynamics simulations and force fields, as both approaches give access to the same time scales as neutron scattering.

To date, there is no complete model taking into account all possible dynamic contributions to the EINS. As a standard, the Gaussian Approximation (GA) is used to extract the MSD of the elastic data. ${ }^{14}$ The limits of the applicability of the models are not always clear and respected such that the way in which they are implemented may seem sometimes arbitrary with full details lacking in many publications. As long as different samples are analyzed in exactly the same way and compared to each other, it may appear to be consistent, but it makes it difficult to compare the results from different samples, spectrometers, and experimental groups. ${ }^{15}$ In particular, using different neutron spectrometers is important for two main reasons. First, each instrument has a specific energy resolution providing access to different dynamical time scales, and second, each instrument has a specific range of accessible scattering angles, which define the spatial information probed. For the first effect, Doster et al. proposed an analysis via elastic resolution spectroscopy in $2001^{16}$ and connected it to temperature scans via a scaling function in 2013. ${ }^{8}$

While the different temporal regimes' information accessed by different neutron spectrometers (different energy resolutions) makes them complementary, the full spatial information accessible on the different instruments due to the range of scattering angles (transformed into momentum transfer Q in reciprocal space) is often redundant, mainly due to theoretical applicability of the GA. Indeed, the GA is in general only valid in a restricted region of $Q$, specifically at low $Q$ values, ${ }^{17}$ since it is neglecting any effects from anharmonicity, heterogeneity, and anisotropy. ${ }^{18}$ To date, limited efforts have been devoted to developing approaches that model EINS data over a wider momentum transfer window, explore the effects of these three aspects, and question whether extra information is contained in the higher $\mathrm{Q}$ regime. Anharmonicity of motions was introduced in a model by Doster et al. ${ }^{2}$ and described in terms of a double-well potential. The introduction of heterogeneity of motions found to be the main reason for the non-Gaussian behavior ${ }^{19}$ has been approached in two ways. In the publications of Becker and $\mathrm{Smith}^{20}$ and more recently Yi et al. ${ }^{21}$ a second term of a series expansion is included to describe the standard deviation of the MSD, whereas the GA uses only the first term of a cumulant expansion. Similar expansions were already proposed earlier by Rahman ${ }^{22}$ and Sköld et $a l .{ }^{23}$ although they did not relate them explicitly to dynamical heterogeneity. The alternative approach consists in describing the heterogeneity by assuming different forms of the distribution. Nakagawa et al. compared a bimodal, exponential, and Gaussian distribution. ${ }^{24}$ Meinhold et al. used a Weibull distribution, ${ }^{25}$ and Peters and Kneller used a gamma distribution ${ }^{26}$ based on the work of Kneller and Hinsen. ${ }^{27}$ Most recently, a bimodal Gaussian distribution was investigated by Vural et al. ${ }^{28}$ Doster also addressed the issue of protein dynamical heterogeneity by suggesting three different approaches to it. $^{29}$ His main conclusion was to say that data can be fitted as successful when heterogeneity is reduced to two kinds of molecular processes, one corresponding to translational and the other to rotational (coming mainly from methyl groups) motions, the latter being non-Gaussian scattering processes. The former models assume, by contrast, a distribution of purely Gaussian motions, which according to Refs. 19 and 30 is also a good approximation when the motions are anharmonic. All the aforementioned models can describe EINS data over an extended Q-range to a greater or lesser extent. In the present study, we will therefore investigate four different models to analyze the same set of EINS data to not only substantiate limits of applicability but also arrive at their advantages and disadvantages by considering data from different instruments and under different experimental conditions. The models to be assessed are GA, Yi et al. (Yi), Peters et al. (PK), and Doster et al. (Do), and a subset is chosen to represent the different approaches.

As the experimental dataset, we choose to measure the MSD of a simple protein powder at different levels of hydration on various instruments. We selected a small commercially available protein: Alpha-lactalbumin (A-L). It is a globular protein (molecular weight of $14.2 \mathrm{kDa}$ ), which regulates lactose synthase and can be found in all mammalian milk. It can strongly bind calcium $\mathrm{Ca}^{2+}$, but also other metal ions like magnesium. The hydration conditions chosen are $\approx 0$ (dry), 0.4 , and $0.8 \mathrm{~g} \mathrm{D}_{2} \mathrm{O} / \mathrm{g}$ protein. Data were collected using three neutron backscattering spectrometers covering a wide temporal range, IN13 at the Institut Laue Langevin (ILL) in Grenoble, France, SPHERES operated by JCNS at the Heinz Maier-Leibnitz Zentrum (MLZ) in Garching, Germany, and OSIRIS at the STFC Rutherford Appleton Laboratory ISIS in Oxfordshire, UK. They correspond to the most common type of neutron backscattering instruments used for EINS experiments. SPHERES and OSIRIS use crystal analyzers that reflect cold neutrons ( $\lambda$ of 6.27 and $6.66 \AA$, respectively) and therefore access a limited Q-range (up to $\left.1.8 \AA^{-1}\right)$, whereas IN13 uses a thermal neutron crystal analyzer ( $\lambda$ of $2.23 \AA$ ), resulting in the largest available Q-range for a neutron backscattering instrument (up to $4.9 \AA^{-1}$ ). In addition, the instruments have different energy resolutions allowing access to motions from a few picoseconds to a few nanoseconds.

The aim of this study is to test the applicability and determine the limitations of four distinct EINS models with respect to different samples and neutron instruments. As we will show, in some cases, clear conclusions can be made, and in others, we simply point out the limitations and let the reader choose the best data analysis method according to priorities imposed by the experiment. 


\section{MATERIALS AND METHODS}

\section{A. Sample preparation}

The sample, $\mathrm{Ca}^{2+}$ depleted A-L, for which all calcium atoms have been purged from A-L, was bought from SigmaAldrich as a lyophilized powder and was used without further purification. According to the supplier, the powder might contain traces of ammonium sulfate or sodium phosphate. Such buffers can contribute to small spurious effects at low temperatures, ${ }^{8}$ but such investigation was beyond the scope of the study. Here, we compared three different levels of hydration, i.e., $\mathrm{h} \approx 0$ (dry), $\mathrm{h}=0.4$, and $\mathrm{h}=0.8$, with $\mathrm{h}$ designating $\mathrm{g} \mathrm{D}_{2} \mathrm{O} / \mathrm{g}$ of dry powder. The dry sample represents the case where only harmonic motions are present up to room temperature. $0.4 \mathrm{~h}$ corresponds to about one or two layers of water on the protein surface, ${ }^{31}$ sufficient to allow for anharmonic motions above the dynamical transition temperature. $0.8 \mathrm{~h}$ represents a gel close to full hydration. In neutron experiments, one often uses $\mathrm{D}_{2} \mathrm{O}$ instead of $\mathrm{H}_{2} \mathrm{O}$ as the solvent since the scattering contribution will be small compared to the very large incoherent scattering contribution from the hydrogens within the protein. ${ }^{32}$ To prepare the hydrated samples, the lyophilized dry protein powder was placed in an open flat aluminum sample holder and in a desiccator over $\mathrm{D}_{2} \mathrm{O}$ and the final hydration level was checked by weighing the sample periodically. When the desired uptake was reached, the sample holder was vacuum sealed with an indium wire. Aluminum is the preferential material for this type of neutron experiments since it is mainly transparent to neutrons, and thus, its contribution to the scattering signal will be negligible. The amount of protein in each sample was close to $100 \mathrm{mg}$. The sample transmissions, as measured on the IN13 spectrometer, were between $92 \%$ and $96 \%$, and therefore, no correction for multiple scattering was applied.

\section{B. Elastic incoherent neutron scattering experiment}

The measurements were carried out on $\mathrm{IN}^{3} 3^{33}$ (data are available in Ref. 34), SPHERES, ${ }^{35,36}$ and OSIRIS ${ }^{37}$ with the characteristic energy resolution $\Delta E_{\text {res }}$ and maximal used momentum transfer, Q, ranges being given in Table I. SPHERES is a reactor-based neutron backscattering spectrometer which uses Si111 analyzer crystals to achieve a sub- $\mu \mathrm{eV}$ resolution, similar to IN16B at the ILL and HFBS at the NIST Center for Neutron Research in Gaithersburg, MD, USA. Due to the instrument design constraints, these spectrometers suffer from slightly degraded energy resolution at the low angles and sometimes lower flux in the first detectors. OSIRIS is a time-offlight near-backscattering neutron spectrometer at a spallation source. It utilizes PG002 analyzer crystals, and while it affords lower energy resolution than SPHERES, it has a larger neutron flux at the sample position and constant $\Delta E$ across the detector bank. Finally, IN13 is a reactor-based neutron backscattering

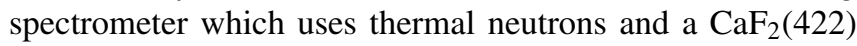
analyzer. This analyzer type results in a much larger $\mathrm{Q}$ range, compared to the OSIRIS and SPHERES instruments, but, consequently, it does suffer worse $\mathrm{Q}$ resolution across the detector bank.

All samples were initially cooled to cryogenic temperatures $(<20 \mathrm{~K})$, and then measurements are taken on warming. At IN13 and SPHERES, the data were collected while applying a continuous heating gradient. At OSIRIS, heating was done in steps of $10 \mathrm{~K}$ or $20 \mathrm{~K}$ and the measurement was then performed at constant temperature once the sample temperature had equilibrated. The data were reduced with LAMP, ${ }^{38}$ Mantid $^{39}$ (v3.11.0), and Slaw (http://apps.jcns.fz-juelich.de/man/slaw.html). Usual data corrections implemented in these packages were applied, including a correction for detector efficiency and subtraction of the empty sample holder. The resulting intensities were normalized to the intensity at the lowest available temperature to correct for any geometrical effects.

\section{Theoretical background and used models}

The atomic motions in a given sample, as measured by neutron spectroscopy, are described by the so-called incoherent dynamic scattering function (DSF) $S_{\text {inc }}(\mathbf{Q}, \omega)$,

$$
S_{\text {inc }}(\mathbf{Q}, \omega)=\frac{1}{2 \pi} \int_{\infty}^{-\infty} I_{\text {inc }}(\mathbf{Q}, t) \exp (-i \omega t) \mathrm{d} t,
$$

where $\mathbf{Q}$ is the momentum transfer, $\omega$ is the energy transfer from the neutron to the sample in units of $\hbar$, and $I(\mathbf{Q}, t)$ is the incoherent intermediate scattering function for one type of atom in the classical limit. It is defined as ${ }^{4}$

$$
I_{\text {inc }}(\mathbf{Q}, t)=\frac{1}{N} \sum_{j=1}^{N}\left\langle\exp \left\{i \mathbf{Q} \cdot \mathbf{r}_{j}(t)\right\} \exp \left\{-i \mathbf{Q} \cdot \mathbf{r}_{j}(0)\right\}\right\rangle,
$$

where $N$ is the number of atoms in the sample, $\mathbf{r}_{j}$ is the position of each individual atom $\alpha$, and \langle\rangle depicts the statistical average of the ensemble. In this study, only $\mathrm{H}$ atoms are considered since they have by far the largest incoherent cross section in a protein. $^{32}$

In the case of confined motion, as is the case in low hydrated powders, the $I_{\mathrm{inc}}(\mathbf{Q}, t)$ tends to a plateau value for $t \rightarrow \infty$,

TABLE I. Characteristics and sample temperatures measured on each instrument. The time window $\Delta t_{\text {res }}$ is calculated using Heisenberg's uncertainty principle $\Delta t_{\text {res }} \cdot \Delta \omega_{\text {res }} \geq \hbar$.

\begin{tabular}{lccccc}
\hline \hline Instrument & Max. used Q range $\left(\AA^{-1}\right)$ & $\Delta \omega_{\text {res }}(\mu \mathrm{eV})$ & $\Delta t_{\text {res }}$ & T range $(\mathrm{K})$ & Heating \\
\hline OSIRIS (ISIS) & $0.30-1.5$ & 25 & $\approx 25 \mathrm{ps}$ & $10-310$ & Steps \\
IN13 (ILL) & $0.49-4.5$ & 8 & $\approx 0.1 \mathrm{~ns}$ & $20-315$ & Gradient \\
SPHERES (MLZ) & $0.27-1.9$ & 0.7 & $\approx 1 \mathrm{~ns}$ & $3-310$ & Gradient \\
\hline \hline
\end{tabular}




$$
\begin{aligned}
\lim _{t \rightarrow \infty} I_{\text {inc }}(\mathbf{Q}, t) & =I_{\text {inc }}(\mathbf{Q}, \infty) \\
& =\frac{1}{N} \sum_{j=1}^{N}\left\langle\exp \left\{-i \mathbf{Q} \cdot \mathbf{r}_{j}\right\}\right\rangle\left\langle\exp \left\{i \mathbf{Q} \cdot \mathbf{r}_{j}\right\}\right\rangle .
\end{aligned}
$$

Here 2 things were assumed: (1) At $t=\infty, \mathbf{r}_{j}(\infty)$ and $\mathbf{r}_{j}(0)$ are statistically independent and (2) $\mathbf{r}_{j}(t)$ is translationally invariant in time such that $\mathbf{r}_{j}(\infty)=\mathbf{r}_{j}(0)=\mathbf{r}_{j}$. Equation (3) can be rewritten using a cumulant expansion of $\left\langle\exp \left\{ \pm i \mathbf{Q} \cdot \mathbf{r}_{j}\right\}\right\rangle$,

$$
\begin{aligned}
I_{\text {inc }}(\mathbf{Q}, \infty)= & \frac{1}{N} \sum_{j=1}^{N} \exp \left\{2 \left[-Q^{2} \rho_{2}\left(\mathbf{n}_{\mathbf{q}}\right)\right.\right. \\
& \left.\left.+Q^{4} \rho_{4}\left(\mathbf{n}_{\mathbf{q}}\right)-Q^{6} \rho_{6}\left(\mathbf{n}_{\mathbf{q}}\right)+\cdots\right]\right\},
\end{aligned}
$$

where $\rho_{i}$ are the moments

$$
\begin{aligned}
\rho_{2}\left(\mathbf{n}_{\mathbf{q}}\right) & =\frac{1}{2 !}\left\langle\left(\mathbf{n}_{\mathbf{q}} \cdot \mathbf{r}_{j}\right)^{2}\right\rangle, \\
\rho_{4}\left(\mathbf{n}_{\mathbf{q}}\right) & =\frac{1}{4 !}\left\{\left\langle\left(\mathbf{n}_{\mathbf{q}} \cdot \mathbf{r}_{j}\right)^{4}\right\rangle-3\left\langle\left(\mathbf{n}_{\mathbf{q}} \cdot \mathbf{r}_{j}\right)^{2}\right\rangle^{2}\right\}, \\
\ldots &
\end{aligned}
$$

$Q=|\mathbf{Q}|$, and $\mathbf{n}_{\mathbf{q}}$ is the unit vector along $\mathbf{Q}$. In energy space, this gives rise to the elastic incoherent structure factor (EISF) $S_{\text {inc }}(\mathbf{Q}, \omega=0)$,

$$
S_{\text {inc }}(\mathbf{Q}, \omega)=\int_{-\infty}^{\infty} \mathrm{d} t \exp (i \omega t) I(\mathbf{Q}, t) R(t)
$$

$$
\begin{aligned}
& S_{\text {inc }}\left(\mathbf{Q}, \omega \approx 0, \Delta \omega_{\text {res }}=\right. \\
& \left.=\int_{-\infty}^{\infty} \mathrm{d} t I(\mathbf{Q}, t) R(t)=A I_{\text {res }}\right) \\
& \qquad \begin{aligned}
S_{\text {inc }}^{\mathrm{n}}\left(\mathbf{Q}, \omega \approx \tau_{\text {res }}\right), \\
=\frac{S_{\text {inc }}\left(\mathbf{Q}, \omega \approx 0, \Delta \omega_{\text {res }}\right)}{S_{\text {inc }}\left(\mathbf{Q}=0, \omega \approx 0, \Delta \omega_{\text {res }}\right)} \\
=I_{\text {inc }}\left(\mathbf{Q}, \tau_{\text {res }}\right) \approx I_{\text {inc }}(\mathbf{Q}, \infty),
\end{aligned}
\end{aligned}
$$

where $R(t)$ is the resolution function of the instrument with a width of $\tau_{\text {res }}=\hbar / \omega_{\text {res }}$ due to which the integration over $I(Q$, $t$ ) is truncated. ${ }^{8,16} \mathrm{~A}$ is a convenient normalization factor to take into account the integration over the instrumental resolution. Here it is important to mention that the plateau value of $t \rightarrow \infty$ is only reached for an ideal instrument with an energy resolution of $\Delta \omega_{\text {res }} \rightarrow 0$. Since a value of zero is impossible to reach, experimentally the value of $S_{\text {inc }}(\mathbf{Q}, \omega \approx 0)$ is dependent on the resolution of the instrument, and therefore to observe different dynamics, one needs to combine experiments performed using different spectrometers. ${ }^{40,41}$ In the following, we suppress the explicit mention of the instrumental resolution, as done in Eq. (8). Additionally, the QENS signal at zero frequency $(\omega=0)$ was neglected.

The Gaussian Approximation (GA) uses only the first nonzero cumulant such that

$$
S_{\mathrm{inc}}^{\mathrm{n}}(Q, \omega \approx 0) \approx \exp \left(-\frac{1}{3} Q^{2}\left\langle r^{2}\right\rangle_{\mathrm{GA}}\right),
$$

where $\left\langle r^{2}\right\rangle$ is the atomic mean square displacement (MSD), which corresponds to the average of the amplitudes of motion of all atoms within the sample. The GA always holds in the case of $Q^{2}\left\langle r^{2}\right\rangle_{\mathrm{GA}} \ll 1$; since then, the higher cumulant terms evaluate to zero, and thus, the GA is exact for some specific cases, i.e., an harmonic solid or an ideal gas. It is by far the most commonly used model in publications about protein dynamics investigated with EINS $^{3}$ and implemented by fitting $\ln [S(Q)]$ vs $Q^{2}$. However, the choice of the Q-range used to fit the data and the value of $\ln [S(Q)]$ at $Q^{2}=0 \AA^{-2}$ is not always specified nor discussed in publications, neither is the limit of $\left\langle r^{2}\right\rangle_{\mathrm{GA}} Q_{\max }^{2} \leq 1$ justified. ${ }^{42}$ Furthermore, sometimes this limit is surpassed and the usage of a larger $Q_{\max }^{2}$ is justified if there is an extended linear behavior of the data points over wider Q-ranges. However a clear rule is not well documented.

It is important to note that the factor of $1 / 3$ in front of the MSD is due to the fact that elastic scattering is time independent and a plateau value for the ISF is reached (which is dependent on the instrument resolution, as mentioned above). For time resolved measurements $[\mathrm{MSD}(\mathrm{t})]$, the factor evaluates to $1 / 6$.

The assumption of the Gaussian approximation for an individual atom $j$ is a good approximation beyond low $\mathrm{Q}$ out to

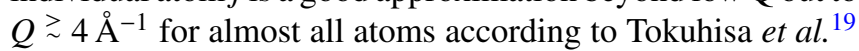
and Vural et al., ${ }^{30}$ as the higher order terms are negligible compared to the $Q^{2}$ term. Moreover, Gaussian heterogeneity is able to describe approximately non-Gaussian (for instance rotational) motions, whose importance is well documented by Liu et al. ${ }^{43}$ The two main reasons, why the GA might not be a valid approximation for an individual atom, are large anharmonic and anisotropic effects. By contrast, Kneller and Chevrot ${ }^{18}$ claim that models could be improved by accounting for anisotropy, but due to the lack of precision of today's available data (neither experimental nor with simulations), additional parameters cannot be fit unambiguously. In the following, we therefore use two heterogeneity models to verify these assumptions.

The first heterogeneity model will be referred to as the Yi model. Applying similar expansions as suggested earlier by Rahman ${ }^{22}$ and Sköld et al., ${ }^{23}$ Becker and Smith ${ }^{20}$ introduced a model beyond the Gaussian approximation within the context of dynamical heterogeneity. Yi et al. ${ }^{21}$ recently proposed it as a "simple correction" of the GA which can be written as

$$
S_{\mathrm{inc}}^{\mathrm{n}}(Q, \omega \approx 0) \approx \exp \left(-\frac{1}{3} Q^{2}\left\langle r^{2}\right\rangle_{\mathrm{Yi}}\right) \cdot\left(1+\frac{Q^{4}}{18} \sigma_{\mathrm{Yi}}^{2}\right)
$$

where $\sigma_{\mathrm{Yi}}$ describes the standard deviation of the $\operatorname{MSD}\left\langle r^{2}\right\rangle_{\mathrm{Yi}}$. It is similar to the expansion of the fourth cumulant term [Eq. (6), second non-zero term] and the same for $\frac{Q^{4}}{18} \sigma_{\mathrm{Yi}}^{2} \ll 1$. The introduction of $Q^{4}$ accounts for the heterogeneity of the motions, and the spread of the MSD is described via $\sigma_{\mathrm{Yi}}$, without assuming any functional form for the distribution. Since it does not contain higher order terms further than $Q^{4}$, its applicability is limited in Q.

The second heterogeneity model that we will consider is that introduced by Peters and Kneller ${ }^{26}$ (referred to as the PK model from here on) which uses a gamma function to describe the distribution of mean square position fluctuations (MSPFs). It is mathematically written as 


$$
\begin{aligned}
S_{\mathrm{inc}}^{\mathrm{n}}(Q, \omega \approx 0) & \approx \frac{1}{\left(1+\frac{Q^{2}\left\langle r^{2}\right\rangle_{\mathrm{MSPF}}}{3 \beta}\right)^{\beta}}, \\
\sigma_{\mathrm{MSPF}} & =\frac{\left\langle r^{2}\right\rangle_{\mathrm{MSPF}}}{\sqrt{\beta}},
\end{aligned}
$$

where $\left\langle r^{2}\right\rangle_{\text {MSPF }}$ describes the mean of the individual MSPF and $\sigma_{\text {MSPF }}$ is its standard deviation. The parameter $\beta$ describes the distribution of the MSPF with a gamma function. In the case of $\beta \rightarrow \infty$, the GA is retrieved. For a constant $\left\langle r^{2}\right\rangle_{\mathrm{MSPF}}$, the heterogeneity increases with decreasing $\beta$. This model poses no Q-limitation since it includes all higher order terms of the cumulant expansion as long as the individual atoms can be described by the Gaussian term alone and under the assumption that heterogeneity is the only reason for the higher order terms.

As can be seen, the standard deviations introduced for the $\mathrm{Yi}$ and PK models are strongly correlated to the respective definitions of the MSD and MSPF, and although a comparison, at least qualitatively, is certainly possible, a detailed study of the additional information they provide is beyond the scope of this paper, where we concentrate on the MSD itself.

The last model used in this study is that introduced by Doster et $a l^{2}$ In the same way as the PK model, it can be used to describe the entire $Q$ range and is based on a doublewell potential model to describe the anharmonicity of atomic motions. In this model, each hydrogen atom can be found in one of the two different harmonic wells, separated by a distance $d$ and by a free energy difference $\Delta G$. The model will be referred to as the Do model from here on and is mathematically expressed as

$$
\begin{aligned}
S_{\text {inc }}^{\mathrm{n}}(Q, \omega \approx 0) \approx & \exp \left(-\frac{1}{3} Q^{2}\left\langle r^{2}\right\rangle_{\mathrm{Do}, G}\right) \\
& \times\left(1-2 p_{12}(1-\operatorname{sinc}(Q d))\right),
\end{aligned}
$$

where the first term with $\left\langle r^{2}\right\rangle_{\mathrm{Do}, G}$ describes the Gaussian contribution to the MSD and the second term describes the two state model. $p_{12}$ is the product of $p_{1}$ and $p_{2}$ which denote the probability of finding an atom in the ground or excited state, respectively, with $p_{2} / p_{1} \propto \exp (-\Delta G / R T)$. The total MSD is defined as

$$
\begin{aligned}
\frac{\left\langle r^{2}\right\rangle_{\text {Do,tot }}}{3} & =-\left(\frac{d \ln [S(Q, \omega=0)]}{d\left(Q^{2}\right)}\right)_{Q=0} \\
& =\frac{\left\langle r^{2}\right\rangle_{\text {Do }, G}}{3}+\frac{p_{12} d^{2}}{3} .
\end{aligned}
$$

In the case of only one well, $p_{12}=0$ or $d=0$ such that the $\mathrm{GA}$ is retrieved.

An alternative way of comparing EINS data is through the evaluation of the elastic neutron intensities summed over all (or a range of) accessible Q-values $I_{\text {sum }}$. In this way, a comparison can be made without using a model. The summed intensities are much less affected by errors, so they give a more accurate overview over the dynamics taking place within the time scales probed by the spectrometer. Nevertheless, in the limit of the GA, it is possible to relate the $I_{\text {sum }}$ to the inverse of the square of the MSD,

$$
\begin{aligned}
I_{\text {sum }} & \stackrel{\mathrm{GA}}{=} \int_{Q_{\max }^{\prime}}^{Q_{\min }^{\prime}} \exp \left(-\frac{1}{3}\left\langle r^{2}\right\rangle^{\prime} Q^{\prime 2}\right) \mathrm{d} Q^{\prime} \\
& \Rightarrow\left\langle r^{2}\right\rangle^{\prime} \stackrel{\mathrm{GA}}{=} \frac{1}{I_{\text {sum }}^{2}} \cdot C^{2},
\end{aligned}
$$

with

$$
C=\frac{\sqrt{3 \pi}}{2}\left[\operatorname{erf}\left(Q_{\max }^{\prime}\right)-\operatorname{erf}\left(Q_{\min }^{\prime}\right)\right]
$$

where $Q^{\prime}=Q / l$ and $\left\langle r^{2}\right\rangle^{\prime}=\left\langle r^{2}\right\rangle / l^{2}$ are the dimensionless quantities and $l$ is chosen as a typical length scale, i.e., $1 \AA$. erf is the error function. ${ }^{44}$ In the limit of experimental precision, the $I_{\text {sum }}$ is discrete and can be evaluated for each measured temperature $T$ through

$$
\begin{gathered}
I_{\text {sum }}(T)=\sum_{i=Q_{\min }}^{Q_{\max }} I_{i}(T), \\
\left\langle r^{2}\right\rangle_{\text {SumI }}(T) \stackrel{\text { GA }}{\propto} \frac{1}{I_{\text {sum }}^{2}(T)} \AA^{2}
\end{gathered}
$$

where $Q_{\min }$ and $Q_{\max }$ are defined by the validity of the GA or given by the instrument.

\section{METHODOLOGY}

In order to compare the models to the experimental data, the resolution broadened $\operatorname{DSF} S_{\text {inc }}(Q, \omega \approx 0)$ is normalized by the measured DSF $S_{\text {inc }}\left(Q, \omega \approx 0, T=\mathrm{T}_{\text {low }}\right)$ at the lowest measured temperature $\mathrm{T}_{\text {low }}$ where the mobility is assumed to be very close to zero,

$$
\frac{S_{\mathrm{inc}}(Q, \omega \approx 0, T)}{S_{\mathrm{inc}}\left(Q, \omega \approx 0, T=\mathrm{T}_{\mathrm{low}}\right)}=S_{\mathrm{norm}}(Q, \omega \approx 0, T) \equiv \operatorname{EI}(Q, T) .
$$

The models are applied to this normalized DSF which for simplicity is labeled as $\operatorname{EI}(Q)$ from now on.

All models were implemented in Python with the help of the LMFIT-package. ${ }^{45}$ A least-squares fitting procedure was used taking into account the error of counting statistics $\epsilon=\sqrt{\text { no of counts }}$. This fitting method minimizes the following formula:

$$
\chi^{2}=\sum_{i=1}^{N} \frac{\left(y_{i}^{\text {data }}-y_{i}^{\text {model }}\right)^{2}}{\epsilon_{i}^{2}}
$$

where $N$ is the total number of data points, $y_{i}^{\text {data }}$ are the experimental data, and $y_{i}^{\text {model }}$ are the values obtained by the fitting model. The reduced value of $\chi_{\text {red }}^{2}=\left(\chi^{2} /\right.$ no of free parameters) can then be used to quantify the quality of the fit.

As described in Sec. II C, all models used in the comparison have different Q-limitations. The PK and Do models can be used to fit the entire available Q-range for all instruments. The PK model will be applied here to all instruments, whereas the Do model is only shown for IN13 as it has the broadest Qrange. The Yi model can also fit a broad Q-range, but in cases of fast decay of the EISF with increasing momentum transfer $\mathrm{Q}$, the expansion of the GA to $\mathrm{Q}^{4}$ is limited as it neglects higher 
order terms. Therefore, we introduce a cutoff to the Yi model, similar to the GA, after which the EISF at high Q-values will not be fitted anymore. To use a consistent Q-range between the three different hydrated A-L samples, the following procedure is used:

First, a lowest Q-value, $\mathrm{Q}_{\min }$, is chosen to be the same for fitting all models to a dataset from a given instrument. Then the maximum $\mathrm{Q}$-value $\mathrm{Q}_{\max }$ is evaluated for the most hydrated sample $(0.8 \mathrm{~A}-\mathrm{L})$ at the highest temperature $\mathrm{T}_{\max }$ since the decay of the EISF with increasing $Q$ is the largest. For the GA, $\mathrm{Q}_{\max }$ is determined as the last $\mathrm{Q}$-value where the fit $\ln [\mathrm{EI}(Q)]$ vs $Q^{2}$ is still linear. The Yi model can describe the entire available Q-range for the OSIRIS and SPHERES instruments. However, for IN13 (up to $4.5 \AA^{-1}$ ), the model reaches its limit as mentioned before and a cut-off value at $\mathrm{Q}_{\max , \mathrm{Yi}}=2.5 \AA^{-1}$ is introduced. It provides the best compromise between including the widest range of $\mathrm{Q}$-values and yet still describing the experimental data satisfactorily for the $0.8 \mathrm{~h}$ sample. The $\mathrm{Q}_{\max }$ evaluated for each model and each instrument was then the same for all samples and temperatures. The consequences due to the limited number of data points in the available Q-range are elaborated in Sec. III B.

Another important point is the analysis of different Qranges which is covered in Sec. III C. A summary of all different Q-ranges evaluated with the GA, Yi, PK, and Do model is found in Table II.

\section{A. The question of the intercept $\operatorname{EI}\left(Q=0 \AA^{-1}\right)$}

All models should start with the same value of the $\operatorname{EI}(Q)$ at zero momentum transfer. As discussed in Sec. III A, the
TABLE II. Different Q-ranges used for the various models and instruments.

\begin{tabular}{|c|c|c|c|c|}
\hline \multirow[b]{2}{*}{ Model } & \multicolumn{4}{|c|}{ Q-range $\left(\AA^{-1}\right)$} \\
\hline & GA & $\mathrm{Yi}$ & PK & Do \\
\hline \multirow[t]{2}{*}{ IN13 } & $0.5-1.7$ & $0.5-2.5$ & $0.5-4.5$ & $0.5-4.5$ \\
\hline & $1.7-4.5$ & \multicolumn{2}{|c|}{$\ldots$} & $\ldots$ \\
\hline \multirow[t]{3}{*}{ SPHERES } & $0.34-0.6$ & \multicolumn{2}{|c|}{$0.34-1.8$} & $\ldots$ \\
\hline & $0.60-1.2$ & \multicolumn{2}{|c|}{$0.60-1.8$} & $\ldots$ \\
\hline & $0.96-1.8$ & \multicolumn{2}{|c|}{$\ldots$} & $\ldots$ \\
\hline
\end{tabular}

OSIRIS

$0.29-1.5$

theoretical value should be 1 for the normalized $\operatorname{DSF} \operatorname{EI}(Q)$. Due to instrumental and experimental effects, like multiple scattering or coherent effects, the starting value is often lower than 1, especially at higher temperatures. For this reason, the value of $\operatorname{EI}\left(Q=0 \AA^{-1}\right)$ is introduced as a fitting parameter $\leq 1$ and its consequences are evaluated here. In most publications about EINS data, the value at $\operatorname{EI}\left(Q=0 \AA^{-1}\right)$ is not clearly defined. On the contrary, in the case of the normally used linear fit of $\ln [\mathrm{EI}(Q)]$ vs $Q^{2}$, only the slope ( $\left.\propto \mathrm{MSD}\right)$ is reported and not the intercept with the y-axis $\left(=\ln \left[\operatorname{EI}\left(Q=0 \AA^{-1}\right)\right]\right)$. This is surprising as will be apparent later in this paper since this value has a strong impact on the resulting MSD value (see Sec. III B). If the GA is used, this value is unique since a linear fit has a global minimum and therefore has only one solution, but in the case of more complex models, a change in the value at $\operatorname{EI}\left(Q=0 \AA^{-1}\right)$ results in a significant variation in MSD. Furthermore, due to the limited experimental information at $\mathrm{Q}$
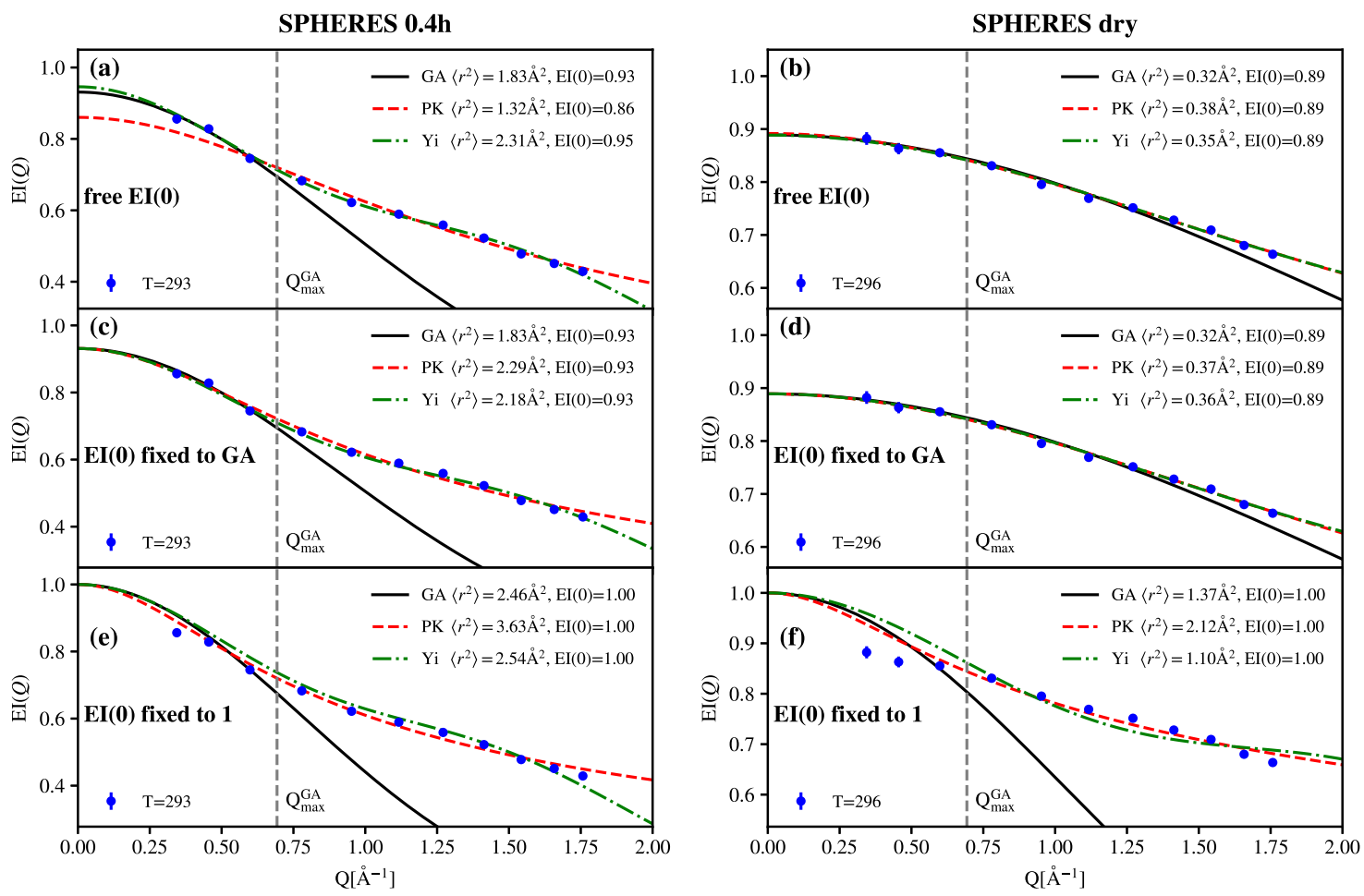

FIG. 1. Example of the effect of $\operatorname{EI}\left(Q=0 \AA^{-1}\right)$ for the SPHERES instrument. The left graphs [(a), (c), and (e)] show an example for A-L with $0.4 \mathrm{~h}$ at $293 \mathrm{~K}$, and the right graphs $\left[(\mathrm{b}),(\mathrm{d})\right.$, and (f)] show an example for dry A-L at $296 \mathrm{~K}$. In graphs (a) and (b), EI $\left(Q=0 \AA^{-1}\right)$ is a free fit parameter. In graphs (c) and (d), $\operatorname{EI}\left(Q=0 \AA^{-1}\right)$ is fixed to the value evaluated with the GA. In graphs (e) and (f), $\operatorname{EI}\left(Q=0 \AA^{-1}\right)$ is fixed to 1 for all models. 
values close to zero given by instrument geometry constraints, and the statistical error of experimental data, it is often not possible to get a unique solution for $\operatorname{EI}\left(Q=0 \AA^{-1}\right)$. Since it is also not possible to fix it to 1 in all cases, we fixed this value, after several trials (see Sec. III A), to be the same for all models and to that obtained by the GA.

To illustrate the importance of the axis intercept $\operatorname{EI}(Q$ $=0 \AA^{-1}$ ), two representative examples are shown in the following. Both datasets are measured on SPHERES at around $295 \mathrm{~K}$. The first one is A-L at 0.4h [Figs. 1(a), 1(c), and 1(e)], and the second one is A-L dry [Figs. 1(b), 1(d), and 1(f)]. In order to qualify the differences between two fits on the same dataset, they are compared in terms of the least-squares error, evaluated by $\chi_{\text {red }}^{2}$ [see Eq. (21)]. The evaluated MSPF/MSD and $\chi_{\text {red }}^{2}$ for the different cases are shown in Table III.

Figure 1(a) shows a visual under-evaluation of the EISF $\operatorname{EI}\left(Q=0 \AA^{-1}\right)$ for the PK model in comparison to the GA if one does not constrain the fit. The gray dashed line indicates the maximum Q-value used for the fit of the GA. The PK and Yi models consider all available Q-values from 0.3 to 1.8 $\AA^{-1}$. The smallest Q-values, 0.3 and $0.46 \AA^{-1}$, are not well described by the fit of the PK model because $\operatorname{EI}\left(Q=0 \AA^{-1}\right)$ $=0.86 \pm 0.03$ is much smaller than the $\operatorname{GA} \operatorname{EI}\left(Q=0 \AA^{-1}\right)$ $=0.93 \pm 0.02$ which fits these points well. By contrast, the Yi model has a slightly higher value of $\operatorname{EI}\left(Q=0 \AA^{-1}\right)=0.95$ \pm 0.01 than the GA, leading to a difference of almost a factor of two in the MSPF/MSD between the PK and Yi model. If the $\operatorname{EI}\left(Q=0 \AA^{-1}\right)$ of these two models is fixed to the value obtained by the GA [see Fig. 1(b)], the lowest two Q-values are now well described by both models, as are the higher Qvalues. The differences in the resulting MSD and $\chi_{\text {red }}^{2}$ for the fixed and free case are shown in Table III. For the PK model, the $\chi_{\text {red, fixed }}^{2}=12$ for the fit where the intercept was fixed is $\approx 20 \%$ larger than that of the free fit result $\chi_{\mathrm{PK} \text {, free }}^{2}=10$. For the Yi model, the $\chi_{\text {red, fixed }}^{2}=5.1$ for the fixed fit is also $\approx 20 \%$ larger than for the free fit $\chi_{\text {red, free }}^{2}=4.0$. In addition, there are big differences in $\chi_{\text {red }}^{2}$ between the PK model and the Yi model even if the fits are visually quite similar for the fixed case [see Fig. 1(b)]. The reason for these is the very small error bars of the counting statistics by which the $\chi^{2}$ is weighted [see Eq. (21)]. The main differences are visible in the Q-range 1.0-1.8 $\AA^{-1}$ where the Yi model follows the data better [see Fig. 1(b)]. However, more important is the effect on the evaluated MSPF of the PK model. In the free case, it is 1.3 $\pm 0.3 \AA^{2}$, while in the fixed case, it is almost a factor of 2 larger with $2.3 \pm 0.2 \AA^{2}$. The changes of the MSD in the Yi model are not as large $\left(2.3\right.$ vs $\left.2.2 \AA^{2}\right)$ since the value $\operatorname{EI}\left(Q=0 \AA^{-1}\right)$ only changed by a small amount. This example is shown to illustrate that even though the least squares chi statistical value may be better for a free fit, the small $Q$-values can be underevaluated for two reasons: (1) The statistical error is smaller for the higher $Q$-values leading to a higher weight on them and (2) the larger $Q$-values can be better described by the models with a lower value at $\operatorname{EI}\left(Q=0 \AA^{-1}\right)$. This under-evaluation of the first Q-values is not only a problem for the PK model. It is not shown here, but it happens for all models describing higher Q-ranges.

Figure 1(c) shows what happens if the $\operatorname{EI}\left(Q=0 \AA^{-1}\right)$ is fixed to 1. Visually, the GA does not describe the first data point well, the PK model is describing the range better, and the Yi model is worse. The resulting MSD/MSPF values are much larger than in the two cases before (see Table III). To emphasis that $\operatorname{EI}\left(Q=0 \AA^{-1}\right)=1$ is not only problematic for hydrated samples, an example for the A-L dry sample at the same temperature is shown in Fig. 1(f). Here the fits show that no model is able to describe the data in the low Q-range. By contrast, they perform well if a free and a fixed value of $\operatorname{EI}\left(Q=0 \AA^{-1}\right)$ to the GA is chosen [see Figs. 1(b) and 1(d)]. It also shows that in this case, fixing the offset is unnecessary since all models evaluate to the same value and that the GA is also closer to the larger Q-values than for the case of $\operatorname{EI}\left(Q=0 \AA^{-1}\right)=1$.

The two examples here show the following: (1) In general, a higher $\operatorname{EI}\left(Q=0 \AA^{-1}\right)$ leads to a higher MSD and (2) the differences between the models can be large if they are allowed to have different values of $\operatorname{EI}\left(Q=0 \AA^{-1}\right)$ and should be kept the same for all models. (3) Fixing $\operatorname{EI}\left(Q=0 \AA^{-1}\right)=1$ is not always possible; therefore when comparing models, the value $\operatorname{EI}\left(Q=0 \AA^{-1}\right)$ should be fixed to the same value, which can be the value evaluated by fitting the GA in the low $Q$-regime where $\left\langle r^{2}\right\rangle Q^{2} \leq 1$. It may be the case that many experimenters use this method to fix the value in their publications, specifically where the authors state that they normalized to $Q=0$, but many do not explain how they achieved that without the knowledge of $\operatorname{EI}\left(Q=0 \AA^{-1}\right)$.

\section{B. Differences between considered Q-ranges within the GA and the influence of statistics}

A second consideration when fitting models to EINS data to extract MSD is the definition of the Q-range to be fitted.

TABLE III. Values for $\chi_{\text {red }}^{2}$ and MSPF/MSD $\left\langle r^{2}\right\rangle$ for the PK and Yi model with the value of $\operatorname{EI}\left(Q=0 \AA^{-1}\right)$ as a free parameter, fixed to the value obtained by the GA and 1 .

\begin{tabular}{|c|c|c|c|c|c|c|c|}
\hline \multirow[b]{2}{*}{ Model } & \multirow{2}{*}{$\begin{array}{c}\text { Example } \\
\operatorname{EI}\left(Q=0 \AA^{-1}\right)\end{array}$} & \multicolumn{3}{|c|}{ A-L $0.4 \mathrm{~h} 294 \mathrm{~K}$} & \multicolumn{3}{|c|}{ A-L dry $296 \mathrm{~K}$} \\
\hline & & Free & Fixed to GA & Fixed to 1 & Free & Fixed to GA & Fixed to 1 \\
\hline PK & $\begin{array}{c}\left\langle r^{2}\right\rangle\left[\AA^{2}\right] \\
\chi_{\mathrm{red}}^{2}\end{array}$ & $\begin{array}{c}1.3 \pm 0.3 \\
10\end{array}$ & $\begin{array}{c}2.3 \pm 0.2 \\
12\end{array}$ & $\begin{array}{c}3.6 \pm 0.5 \\
17\end{array}$ & $\begin{array}{c}0.38 \pm 0.05 \\
1.1\end{array}$ & $\begin{array}{c}0.37 \pm 0.02 \\
0.98\end{array}$ & $\begin{array}{c}2.1 \pm 0.4 \\
9.7\end{array}$ \\
\hline Yi & $\begin{array}{c}\left\langle r^{2}\right\rangle\left[\AA^{2}\right] \\
\chi_{\text {red }}^{2}\end{array}$ & $\begin{array}{c}2.3 \pm 0.1 \\
4.0\end{array}$ & $\begin{array}{c}2.2 \pm 0.1 \\
5.1\end{array}$ & $\begin{array}{c}2.6 \pm 0.2 \\
28\end{array}$ & $\begin{array}{c}0.35 \pm 0.03 \\
1.2\end{array}$ & $\begin{array}{c}0.36 \pm 0.02 \\
1.06\end{array}$ & $\begin{array}{c}1.1 \pm 0.2 \\
29\end{array}$ \\
\hline
\end{tabular}



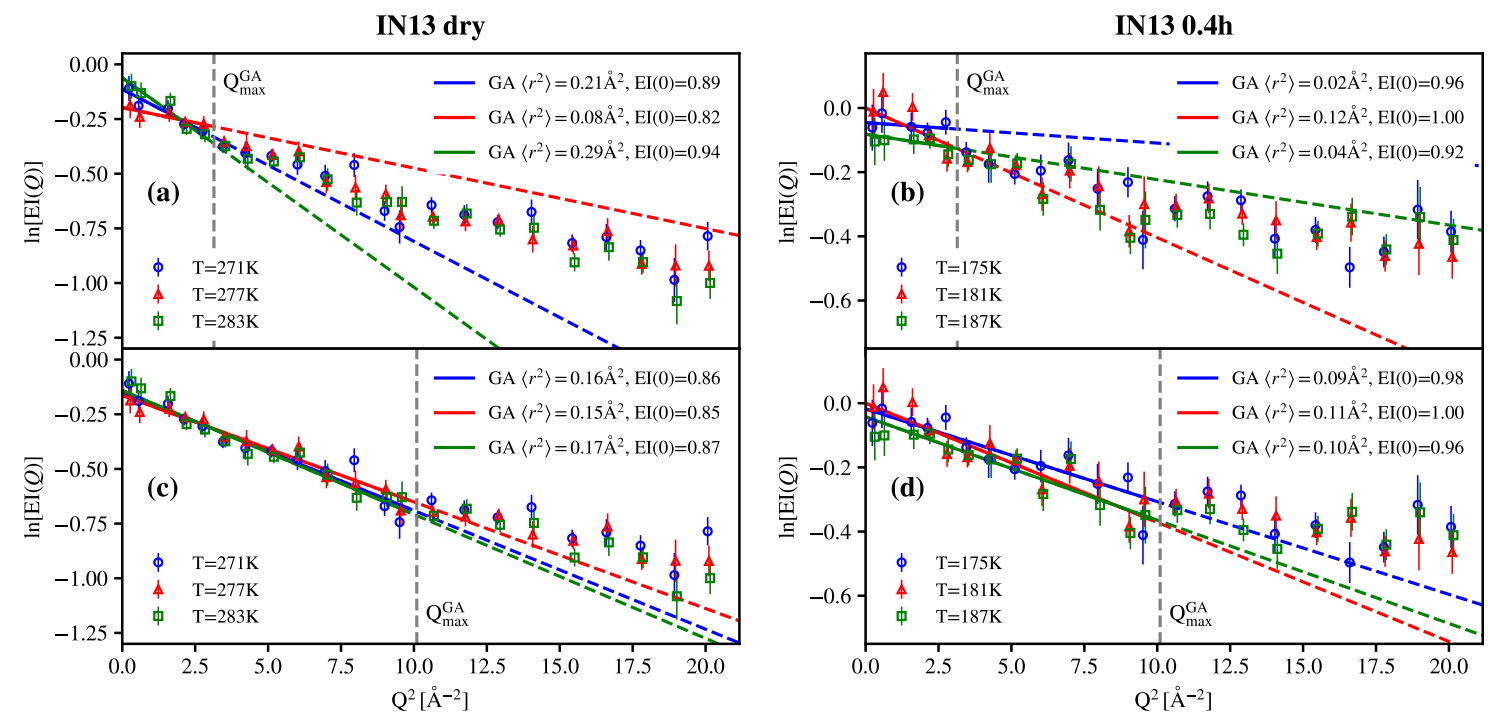

FIG. 2. Effect of statistics on the value of the $\operatorname{EI}\left(Q=0 \AA^{-1}\right)$ and the MSD evaluated with the GA model. For the dry A-L, an example is shown between 271 $\mathrm{K}$ and $283 \mathrm{~K}$ taken on IN13: (a) If we consider only the low Q-range (gray line), the results differ from 0.08 to $0.29 \AA^{2}$ which explains the drop of the MSD at $277 \mathrm{~K}$ shown in Fig. 5(a). (c) Considering Q-values within the validity of the GA model up to $\mathrm{Q}_{\max }^{2}=10 \AA^{-2}$ gives similar results for the MSD since the low Q-values are less weighted. [(b) and (d)] Similar example for the $0.4 \mathrm{~h} \mathrm{A-L} \mathrm{between} 175 \mathrm{~K}$ and $187 \mathrm{~K}$ : (b) low Q-range for GA (d) $\mathrm{Q}_{\max }^{2}=10 \AA^{-2}$ (gray line).

Typically this is vague and deserves further consideration. Many authors in publications cite the criterion defining the upper value $Q_{\max }$ and some discuss arguments why it is reasonable to surpass this limit. ${ }^{46}$ This becomes important because even the value of $\operatorname{EI}\left(Q=0 \AA^{-1}\right)$ for the $\mathrm{GA}$, which will be taken in the following as the starting value for all models, depends on the chosen Q-range and the statistical error of each measured intensity data point. This can be best shown through data collected on the backscattering spectrometer IN13 at the ILL. In order to compare accurately the differences of the MSD of similar proteins, usually the same Q-ranges are chosen for the fit over all temperatures.

Figure 2 illustrates two problems if a limited Q-range is chosen. First Figs. 2(a) and 2(b) show fits to the GA which include data points up to $\mathrm{Q}_{\max }^{2}=2.8 \AA^{-2}$, evaluated as the largest Q-range for the A-L sample from the highest temperature (in total, 5 distinct Q-values with a statistical error of around 5\%-10\%). This region is similar to the Q-range of the other two spectrometers SPHERES and OSIRIS. By fitting only the very first few points, where the GA is strictly valid, large differences result in the MSD even over a temperature range of just $10 \mathrm{~K}$; see Figs. 2(a) and 2(b) for the dry and 0.4h A-L, respectively. Within the statistics, the intensities
$\operatorname{EI}(Q)$ at a given momentum transfer are almost the same for all temperatures, but due to the variation in absolute height of the first 5 Q-values, the resulting MSDs can vary by a factor of 3 (from 0.08 to $0.29 \AA^{2}$ ) [see Fig. 2(a)] or even 5 [from 0.02 to $0.12 \AA^{2}$ ] [see Fig. 2(b)]. On the contrary, if we extend the Q-range and assume the validity of the GA until $\mathrm{Q}_{\max }^{2}=10 \AA^{-2}$ at all temperatures, a region that still reasonably corresponds to a linear fit region and is just slightly larger than the theoretically proposed limit $\left\langle r^{2}\right\rangle_{\mathrm{GA}} \mathrm{Q}_{\max }^{2} \leq 1$, then the resulting MSD is almost the same for all models within error bars: Fig. 2(c), 0.15-0.17 $\AA^{2}$, and Fig. 2(d), 0.09$0.11 \AA^{2}$. A summary of the obtained $\left\langle r^{2}\right\rangle$ and $\chi_{\text {red }}$ is shown in Table IV.

This example shows two important aspects of using the largest available Q-range: (1) A larger Q-range results in more precise and consistent results since we can include more data points and (2) it also leads, in general, to different $\operatorname{EI}(Q=0$ $\AA^{-1}$ ) and MSD values, even when both should give the same result since they are still in the limit of the GA. Therefore, it is important to include the highest possible Q-range to be as precise as possible, but also to stick with the same $\mathrm{Q}$ ranges to compare data accurately.

TABLE IV. Values for $\chi_{\text {red }}^{2}$ and MSD $\left\langle r^{2}\right\rangle$ for the GA model with $Q_{\max }^{2}=2.8 \AA^{-2}$ and $Q_{\max }^{2}=10 \AA^{-2}$.

\begin{tabular}{|c|c|c|c|c|c|c|c|}
\hline \multirow[b]{2}{*}{$Q_{\max }^{2}\left[\AA^{-2}\right]$} & \multirow{2}{*}{$\begin{array}{c}\text { Example } \\
\mathrm{T}(\mathrm{K})\end{array}$} & \multicolumn{3}{|c|}{ A-L dry } & \multicolumn{3}{|c|}{ A-L $0.4 \mathrm{~h}$} \\
\hline & & 271 & 277 & 283 & 175 & 181 & 187 \\
\hline 2.8 & $\begin{array}{c}\left\langle r^{2}\right\rangle\left[\AA^{2}\right] \\
\chi_{\text {red }}^{2} \\
\end{array}$ & $\begin{array}{c}0.21 \pm 0.04 \\
0.38\end{array}$ & $\begin{array}{c}0.08 \pm 0.03 \\
0.21\end{array}$ & $\begin{array}{c}0.29 \pm 0.06 \\
0.92\end{array}$ & $\begin{array}{c}0.02 \pm 0.04 \\
0.30\end{array}$ & $\begin{array}{c}0.12 \pm 0.04 \\
1.3\end{array}$ & $\begin{array}{c}0.04 \pm 0.04 \\
0.24\end{array}$ \\
\hline 10 & $\begin{array}{c}\left\langle r^{2}\right\rangle\left[\AA^{2}\right] \\
\chi_{\mathrm{red}}^{2}\end{array}$ & $\begin{array}{c}0.16 \pm 0.02 \\
0.98\end{array}$ & $\begin{array}{c}0.15 \pm 0.01 \\
0.48\end{array}$ & $\begin{array}{c}0.17 \pm 0.02 \\
1.1\end{array}$ & $\begin{array}{c}0.09 \pm 0.02 \\
0.75\end{array}$ & $\begin{array}{c}0.11 \pm 0.01 \\
0.96\end{array}$ & $\begin{array}{c}0.10 \pm 0.02 \\
0.75\end{array}$ \\
\hline
\end{tabular}




\section{Two regimes-high Q-range}

As already mentioned, the GA model is strictly valid for $\mathrm{Q} \rightarrow 0 \AA^{-1}$, but some experimental and instrumental issues arise specifically at low $Q$ values. We can have multiple scattering ${ }^{47}$ or non-negligible coherent contributions which result in $\mathrm{EI}(0)<1$, detectors of different resolutions for low momentum transfers as is the case for SPHERES ${ }^{48}$ and the very similar instruments IN16B and HFBS. By contrast, IN13 and OSIRIS do not suffer from detector resolution effects. But in general, the counting statistics are also worse at lower Q-values.

To illustrate this, an example for each instrument and hydration between 305 and $310 \mathrm{~K}$ is shown in Fig. 3 as plots of $\ln [\operatorname{EI}(Q)]$ vs $Q^{2}$. As can be seen, the data appear to have different linear regimes. For IN13 and SPHERES, a second linear regime at high $\mathrm{Q}$ is clearly visible, whereas OSIRIS shows only one. The reason is the different dynamical processes which are visible at different time scales (see Sec. II B). On SPHERES, we also note that the first two Q-values used at 0.34 and $0.45 \AA^{-1}$ are clearly higher for the hydrated samples, which is probably due to a slightly reduced resolution of these two detectors. ${ }^{48}$ Therefore, two different low Q regimes are fitted for SPHERES, the first three detectors, including the two Q detectors with lower resolution $\left(0.34-0.60 \AA^{-1}\right)$ and then the next four detectors after these two detectors $\left(0.60-1.2 \AA^{-1}\right)$. To factor out effects at low $\mathrm{Q}$ and to evaluate the information that can be obtained in the second linear regime, the GA is also fitted to high $\mathrm{Q}$ values, even though it strictly falls out of $Q^{2}\left\langle r^{2}\right\rangle_{\mathrm{GA}} \ll 1$. A similar approach was taken in previous publications of IN13 data (e.g., in Ref. 49 or Ref. 50). Figure 3 illustrates using dashed lines all the different linear regimes fitted in this paper (for Q-ranges, see Table II).

In addition to the second linear regime at high Q-values, the ordering between the different instruments, and therefore resolution, can be clearly observed; the break between regions of linearity is moving to smaller momentum transfers with increasing resolution.

\section{RESULTS AND DISCUSSION}

In the following, we discuss the validity of the aforementioned models in more detail comparing to datasets from the three spectrometers IN13, SPHERES, and OSIRIS, in turn. In addition, we show and discuss the summed intensities versus MSPF for the three instruments and hydration levels.

\section{A. The instrument IN13}

The Q-ranges used for IN13 data evaluation are given in Table II. Representative fits are shown at three different temperatures for the A-L at $0.4 \mathrm{~h}$ and $0.8 \mathrm{~h}$ in Figs. 4(a) and 4(b), respectively. All models describe the data points well within their specific Q-ranges. At high temperatures for the $0.8 \mathrm{~h}$ sample, the Yi model follows the general behavior of the experimental data but does not fit as well as the other models. For these data, a lower $\mathrm{Q}_{\max }^{\mathrm{Yi}}$ would be needed to obtain better agreement. However, a smaller Q-range would not include much more $\mathrm{Q}$ information than that already considered using the GA model. For reasons already explained since we want to compare all samples within the same Q-range, we stick to $\mathrm{Q}_{\max }^{\mathrm{Yi}}=2.5 \AA^{-1}$ which is the best compromise between including the largest Q-values possible and a good description of the data with a given model.

The MSD results of the fits to the elastic, normalized DSF $\mathrm{EI}(Q)$ are shown in Fig. 5. Figure 5(a) shows the difference between the GA, PK, and Yi model for the dry protein. The differences between the models are very small, and all models show a similar behavior. The same plot is shown for 0.4 hydration in Fig. 5(b). Here, the differences between the models are also small, but overall, the PK and Yi models give
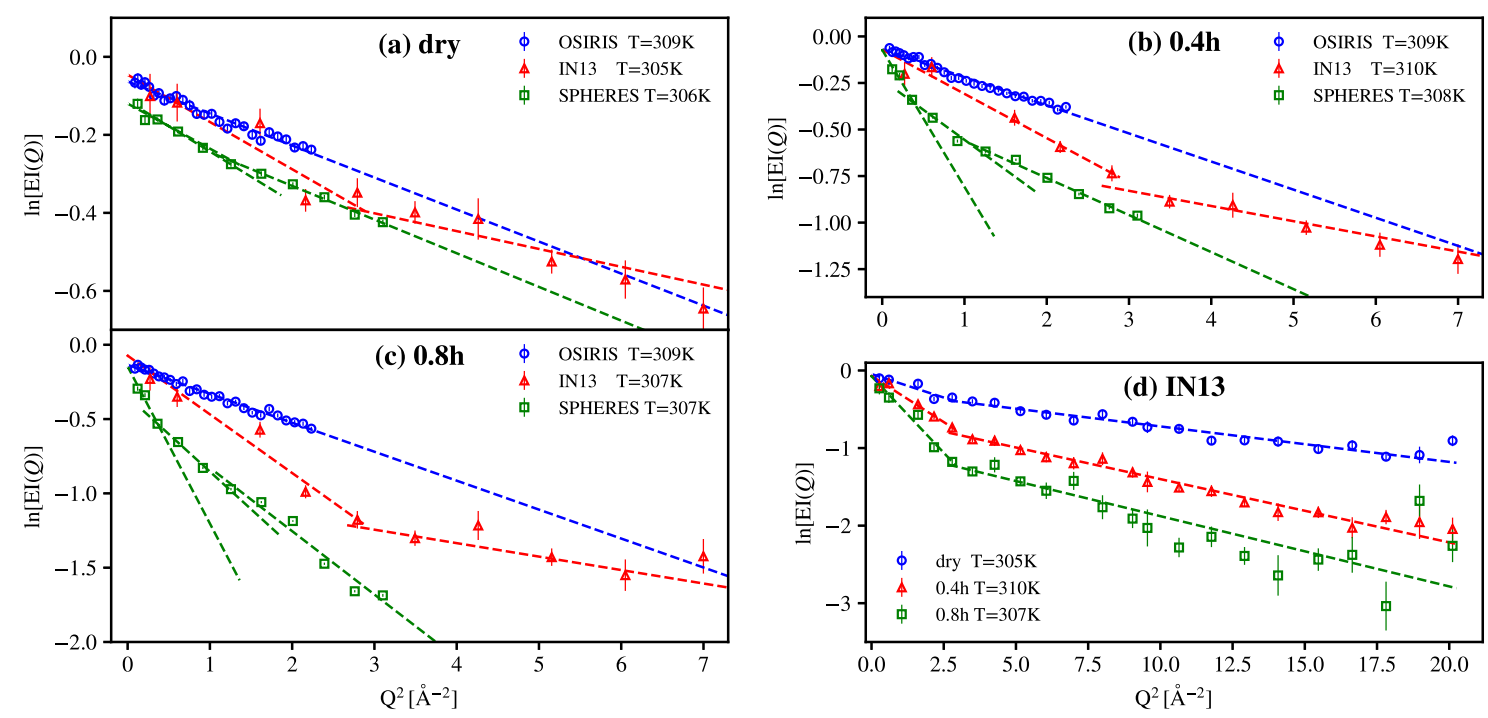

FIG. 3. Normalized raw data $\ln [\operatorname{EI}(Q)]$ vs $Q^{2}$ for the three different hydration levels [(a)-(c)] and the three different instruments, OSIRIS (blue), IN13 (red), and SPHERES (green). The dashed lines indicate linear fits to the data in their respective range. For OSIRIS, at all hydration levels, mainly one linear regime is visible. For IN13, clearly two regimes are visible, separated around $3 \AA^{-2}$. For SPHERES, two low $\mathrm{Q}$ regions and one high $\mathrm{Q}$ region can be identified. (d) shows the entire Q-range of IN13 and the linearity of the second regime up to $20 \AA^{-2}$. 

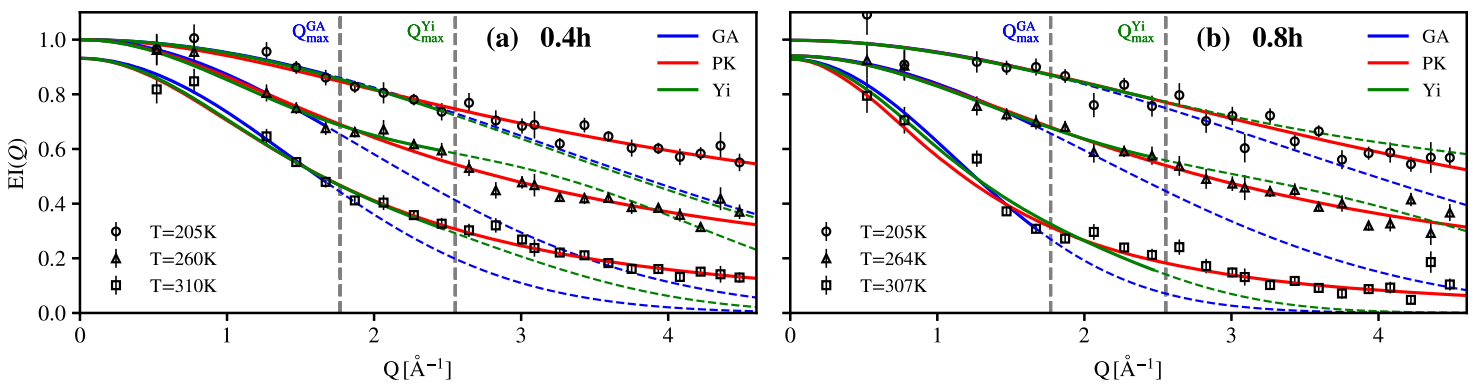

FIG. 4. Representative fits for the three models at three different temperatures for IN13 data. (a) corresponds to $0.4 \mathrm{~h}$ and (b) corresponds to $0.8 \mathrm{~h}$. The vertical gray lines indicate the $\mathrm{Q}_{\max }$ used for each model: $0.5-1.7 \AA^{-1}$ for GA and $0.5-2.5 \AA^{-1}$ for Yi.
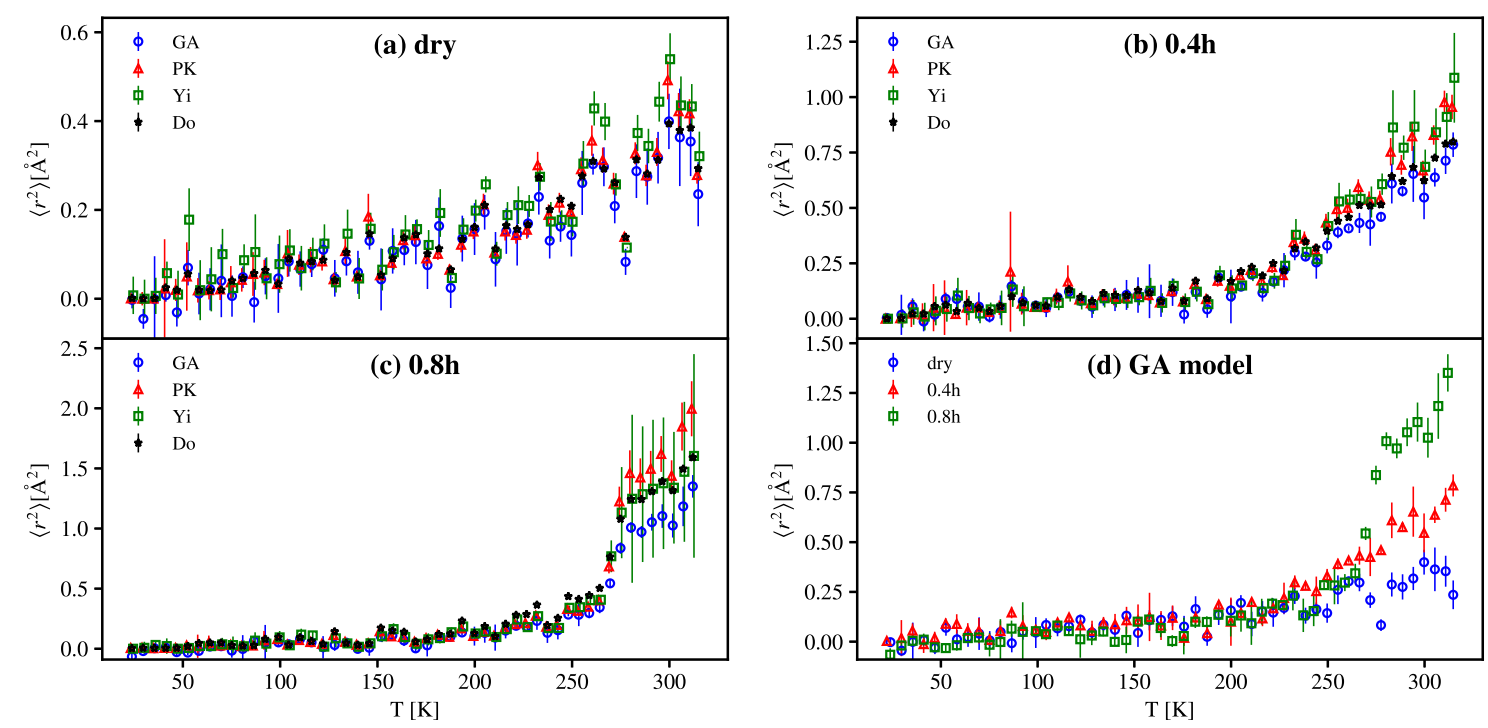

FIG. 5. MSD values extracted from the GA, PK, Yi, and Do models for dry, 0.4, and 0.8 hydrated A-L depleted samples [(a)-(c)]; IN13 data. (d) shows the MSD of all three hydration levels evaluated with the GA model.

rise to slightly higher MSPF/MSD values due to the inclusion of higher $Q$ values. At 0.8 hydration [see Fig. 5(c)], the MSPF/MSD values are clearly higher at high temperatures for both models. Both hydrated samples show an increase in the MSD at around $230 \mathrm{~K}$ compared to the dry protein as expected at the dynamical transition temperature, ${ }^{2}$ commonly observed in hydrated proteins. For the $0.8 \mathrm{~h}$ sample, around $270-280 \mathrm{~K}$, a very steep increase in the MSD is visible that can be attributed to the melting point of heavy water at $278 \mathrm{~K}$. Probably for 0.8 hydration, some free water exists that can freeze and therefore inhibit the motion of the protein. Therefore, the MSD shows an abrupt rise at the melting point. After $280 \mathrm{~K}$, the increase in the MSD for all models is similar to the $0.4 \mathrm{~h}$ sample, but the absolute values are higher. The MSD for the $0.8 \mathrm{~h}$ sample is higher than for the $0.4 \mathrm{~h}$ sample since the higher hydration decreases the crowding in the sample and thus allows more motions. For both hydrated samples, the error bars of the GA are smaller than for the other models. This gives the illusion of a higher accuracy, which is only due to the cut-off effect and to the exclusion of certain amplitudes. In the case of the Yi model at 270-320 K, very large errors are evaluated since the fit is not describing the data as well as for the other cases [e.g., see Fig. 4(b) at $307 \mathrm{~K}$ ]. This can be confirmed by the higher $\chi_{\text {red }}^{2}$ shown in Fig. 6.
Finally, we compare the performance of the models that include heterogeneity to the double well potential model of Doster et al. that takes into account anharmonicity. Since IN13 has by far the largest available Q-range, the Doster model is only evaluated on this instrument to show that it also gives good results. Figures 5(a)-5(c) show the results of $\left\langle r^{2}\right\rangle_{\text {Do,tot }}$ defined

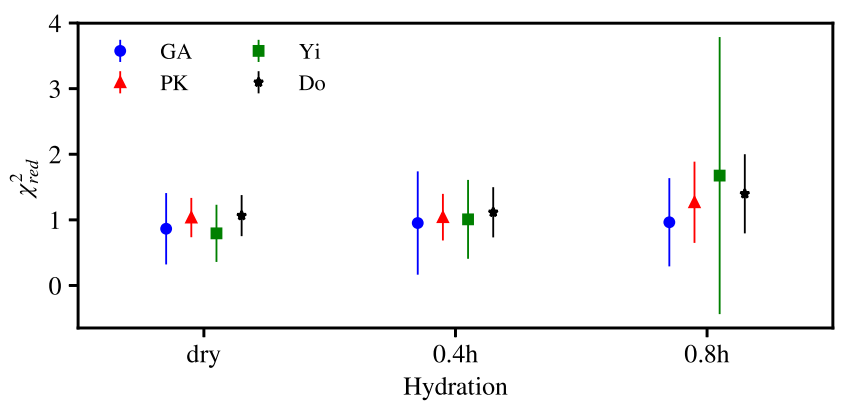

FIG. 6. Reduced $\chi_{\text {red }}^{2}$ for the fits, averaged over all temperatures. It is important to note that the reduced $\chi_{\text {red }}^{2}$ values are calculated with the respective Q-values used for the fit. Therefore, the GA and Yi models take less values into account. All four models have a $\chi_{\text {red }}^{2}$ value around 1 , but the value for the Yi model at 0.8 hydration is around 2 and has a much bigger standard deviation. The reason for this was already mentioned in the results of the evaluated MSD. The fit of the Yi model is indeed worse at high temperatures and therefore also its $\chi_{\text {red }}^{2}$. 
in Eq. (15). They compare rather well with the results from the other three models. The evaluated values for the enthalpy $\Delta G=6.1 \mathrm{~kJ} \mathrm{~mol}^{-1}$ and $\mathrm{d}=1.7 \AA(0.4 \mathrm{~h})$ are similar to values found for myoglobin at $0.38 \mathrm{~h}\left(\Delta G=12 \mathrm{~kJ} \mathrm{~mol}^{-1}, \mathrm{~d}=1.5 \AA\right) .^{2}$ In Fig. 6, the reduced $\chi_{\text {red }}^{2}$ for the different fits averaged over the entire temperature range are shown.

Overall the results on IN13 lead to the conclusion that for the dry A-L, all models are equal since the system is still close to a harmonic system (no dynamical transition visible) and the heterogeneity does not influence the evaluated MSD. When the hydration increases, the anharmonicity grows and quantitative differences can be seen between the models. Especially at 0.8 hydration, the three models diverge at high temperatures, showing the influence of the heterogeneity to the MSD. The quality of the fits is similar for all three models in their respective Q-range. Only in the case of the Yi model, it is worse at high temperatures for the A-L at $0.8 \mathrm{~h}$.

\section{IN13—only high Q-range}

Taking advantage of the fact that IN13 covers a wide Qrange, we follow on from the discussion in Sec. III C to now consider fitting the high Q-range alone using the GA model, to evaluate the effect on the dynamical transition temperature and the MSD. Specifically, we fit the Q-range $1.7-4.5 \AA^{-1}$. The result is shown in Fig. 7 and can be compared to the GA at low Q-values in Fig. 5(d). The absolute value of the MSD from the high Q-range is lower by up to a factor of 5 for the $0.8 \mathrm{~h}$ sample. This is a huge effect, but might be reasonable when separating motions of large and small amplitudes, for instance, atoms of the side chains of the amino acids and fluctuations within the backbone of the amino acids. The relative change in the MSD with increasing temperature between the different hydrations is however similar. All hydrations follow the same trend until $220 \mathrm{~K}$ and then deviate from each other. In the high Q-range, the $0.4 \mathrm{~h}$ and $0.8 \mathrm{~h}$ curves are then superposed until the second splitting due to the melting of heavy water, whereas for the small Q-range, the MSD of the higher hydrated sample lies even below the $0.4 \mathrm{~h}$ sample.

In the paper of Combet and Zanotti, ${ }^{51}$ the authors study a protonated protein hydrated in $\mathrm{D}_{2} \mathrm{O}$ and the same protein in a per-deuterated form hydrated in $\mathrm{H}_{2} \mathrm{O}$ on two different instruments, IN13 and the spectrometer MIBEMOL with a resolution of $\approx 140 \mu \mathrm{eV}$. The corresponding short time window of $\approx 10 \mathrm{ps} \mathrm{s}$ reveals a weak dynamical transition, observed for both the protein and its hydration water. By contrast, the larger

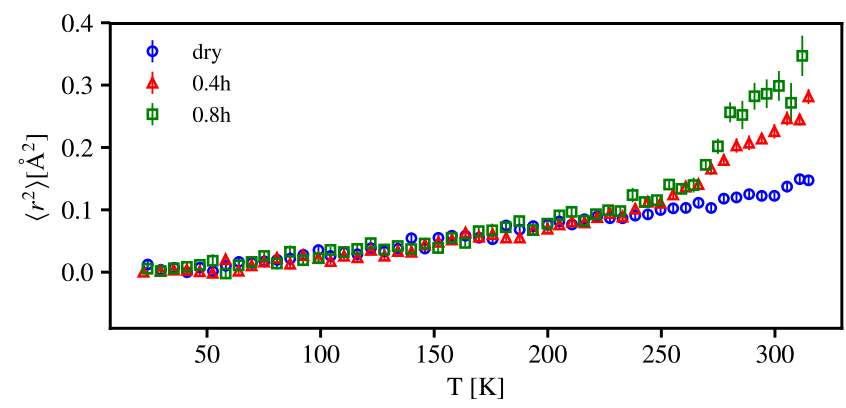

FIG. 7. MSD for all three hydrations evaluated with the GA model at a high Q range (1.7-4.5 $\left.\AA^{-1}\right)$; IN13 data. time window of IN13 permits a separation of the experimental data into large and local motions with a crossover at around $1.2 \AA^{-1}$; clear differences in the motions of water and protein molecules are being visible since larger amplitude motions can be probed at low $\mathrm{Q}$. Their approach of using H/D-contrast allows direct evidence of two populations of motion. Our findings without contrast variation confirm that a division in two population is indeed reasonable.

Another approach in this same line of thought involves fitting the whole Q-range using a bimodal fitting model, where $\operatorname{EI}(Q) \approx p_{1} \exp \left(-\left\langle r^{2}\right\rangle_{\text {large }} Q^{2}\right)+p_{2} \exp \left(-\left\langle r^{2}\right\rangle_{\text {local }} Q^{2}\right)$, with $p_{1}+p_{2}=1$. It assumes the presence of two well-separated MSDs defined within the GA $\left.\left\langle r^{2}\right\rangle_{\text {large }}\right\rangle\left\langle r^{2}\right\rangle_{\text {local }}$ in the sample, as proposed by Nakagawa et al. ${ }^{24}$ This approach works well for the protein staphylococcal nuclease with an instrumental resolution of $1 \mathrm{~m} \mathrm{eV}$, obtaining $\left\langle r^{2}\right\rangle_{\text {large }} \approx 0.7 \AA^{2}$ and $\left\langle r^{2}\right\rangle_{\text {local }} \approx 0.15 \AA^{2}$ at $300 \mathrm{~K}$.

In conclusion, our results suggest that by analyzing the high Q range only on an instrument like IN13, one can access local motions in the proteins, which give rise to small amplitudes of motion, but which follow quite closely the temperature and hydration behavior of the large amplitudes in the case of hydrated powders.

\section{B. The instrument SPHERES}

For the SPHERES spectrometer, the used Q-range is $0.34-1.8 \AA^{-1}$. The first two detectors at $Q=0.34$ and $Q=0.45 \AA^{-1}$ have a lower energy resolution. ${ }^{48}$ To see the influence of this resolution effect, two different low Q-ranges were fitted in which the GA is still valid, i.e., $\ln [\mathrm{EI}(Q)]$ vs $Q^{2}$ linear for all temperatures. The first low Q-range only includes the first three available Q-values $0.34-0.6 \AA^{-1}$ (see Sec. IV B 1), whereas the second low Q-range excludes the first two Q-values with lower resolution, corresponding to a Q-range of 0.6-1.2 $\AA^{-1}$ (see Sec. IV B 2). In both cases, the PK and Yi models are fitted to the same Q-values as the respective fit of the GA and using in addition the larger available Q-values until $1.8 \AA^{-1}$. A third Q region is fitted to only high Q-values, as described in Sec. III C. There only the GA was used in the Q-range $0.95-1.8 \AA^{-1}$, neglecting the first four Q-values.

\section{Low Q-range (GA, 0.34-0.6 $\AA^{-1}$ )}

In Figs. 8(a) and 8(b), representative fits for three different temperatures are shown for the $0.4 \mathrm{~h}$ and $0.8 \mathrm{~h} \mathrm{~A}-\mathrm{L}$ samples. The fits show that it is not possible to include more Q-values for the GA if the small Q-values should still be well described by the fit. A comparison to a larger Q-range which does not describe the lowest two Q-values is shown in Sec. IV B 2. The SPHERES spectrometer has ten times better resolution than IN13 such that larger motions are included and the resulting MSD becomes larger, leading to a lower Q-range accessible by the GA. These larger motions are possibly the movements of the side chains of A-L. Also small differences are visible between the samples in comparison to IN13.

The results of the fits of the $\operatorname{EI}(Q)$ are shown in Fig. 9 in the same way as for IN13. For all samples, a linear increase of the MSD/MSPF is visible until $200 \mathrm{~K}$ where the dynamical transition sets in. 

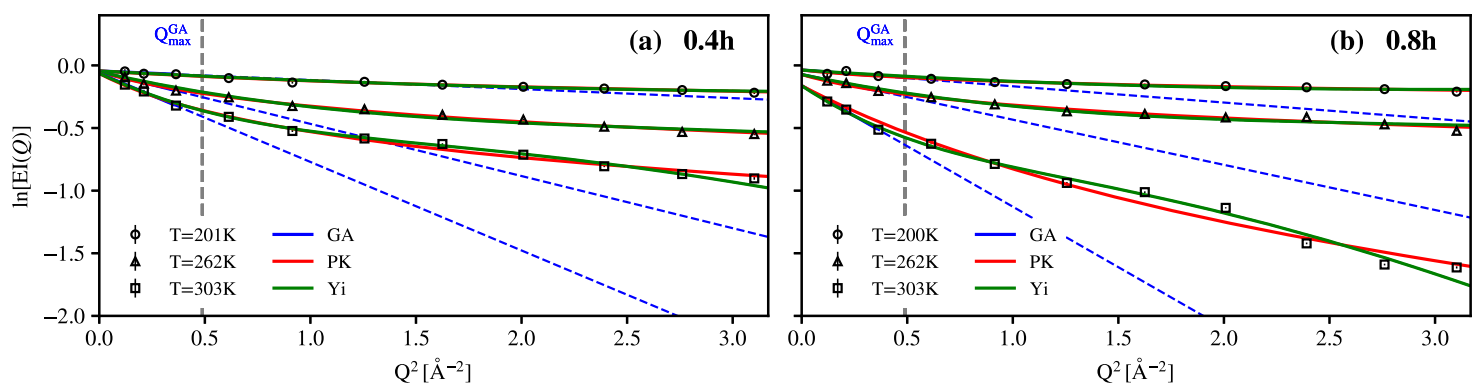

FIG. 8. Representative fits for the three models at three different temperatures for SPHERES data. (a) corresponds to 0.4h, and (b) corresponds to $0.8 \mathrm{~h}$. The vertical gray line indicates $\mathrm{Q}_{\max }=0.6 \AA^{-1}$ used for the GA.

For the dry sample, the evaluated MSD is very noisy which can be explained by the low statistics implied in using only the first three Q-values. Higher Q-values could be included, but in order to compare the same Q-ranges between the different hydrations, we stick to the Q-range evaluated for the highest hydration (for a larger Q-range, see Sec. IV B 2). The problem with using the first three data points only is emphasized by the small decay of the $\operatorname{EI}(Q)$ for the dry sample. The Yi and PK models fit the data well and with a similar goodness, resulting in similar values of MSD/MSPF, and with a much better accuracy than the GA since they are including all experimental data points.

At 0.4 hydration, the dynamical transition is visible around $200 \mathrm{~K}$. The MSPFs/MSDs of the PK and Yi model are higher than the MSDs of the GA model following the trends seen on IN13. At 0.8 hydration, two changes in the slope are visible at $\sim 200 \mathrm{~K}$ and $\sim 270 \mathrm{~K}$. The first change of the slope is again attributed to the dynamical transition and the second is attributed to the melting of free heavy water which enhances the movements of the protein. The highest MSD/MSPF values are around $3.5 \AA^{2}$. The PK model has the same MSPF as the MSD of the GA, whereas the Yi model has higher MSD at temperatures above $270 \mathrm{~K}$. This coincides again with the melting point of $\mathrm{D}_{2} \mathrm{O}$. The MSD from 270 to $280 \mathrm{~K}$ increases in the Yi model by almost $1.5 \AA^{2}$. This transition is smoother for the two other models. On the other hand, after the jump, the increase of the MSD in the Yi model is much slower in comparison to the other models. Therefore, at $310 \mathrm{~K}$, the 3 models reach a similar value in MSD/MSPF. The jump of the Yi model can be explained by the counterbalancing between the $\mathrm{Q}^{2}$ and $\mathrm{Q}^{4}$ term [see Eq (11)], which results likely into a mathematical, but not a physical solution. Also the reduced $\chi_{\text {red }}^{2}$ statistics indicate that the fitting of the $\mathrm{PK}$ and Yi model for 0.8 hydrated samples is much worse than for the dry and 0.4 hydration samples (data not shown).

\section{Low Q-range (GA, 0.6-1.2 $\AA^{-1}$ )}

In Sec. IV B 1, the GA was only fitted to the first three Qvalues $\left(0.35-0.6 \AA^{-1}\right)$ to take into account the limit of validity given in Eq. (10). This leads to a good description of the data in the low Q-range, but also to a high statistical error. In addition, as stated before, the resolution is larger for the first two low $\mathrm{Q}$ detectors in comparison to the other detectors. If the GA is fitted in the range of $0.6-1.2 \AA^{-1}$, it leads to a more consistent linear fit with a smaller error. Examples for the fits are shown in Figs. 10(a) and 10(b) as before. The resulting MSDs are shown in Fig. 11, together with the Yi and PK models using the Q-range $0.34-1.8 \AA^{-1}$.
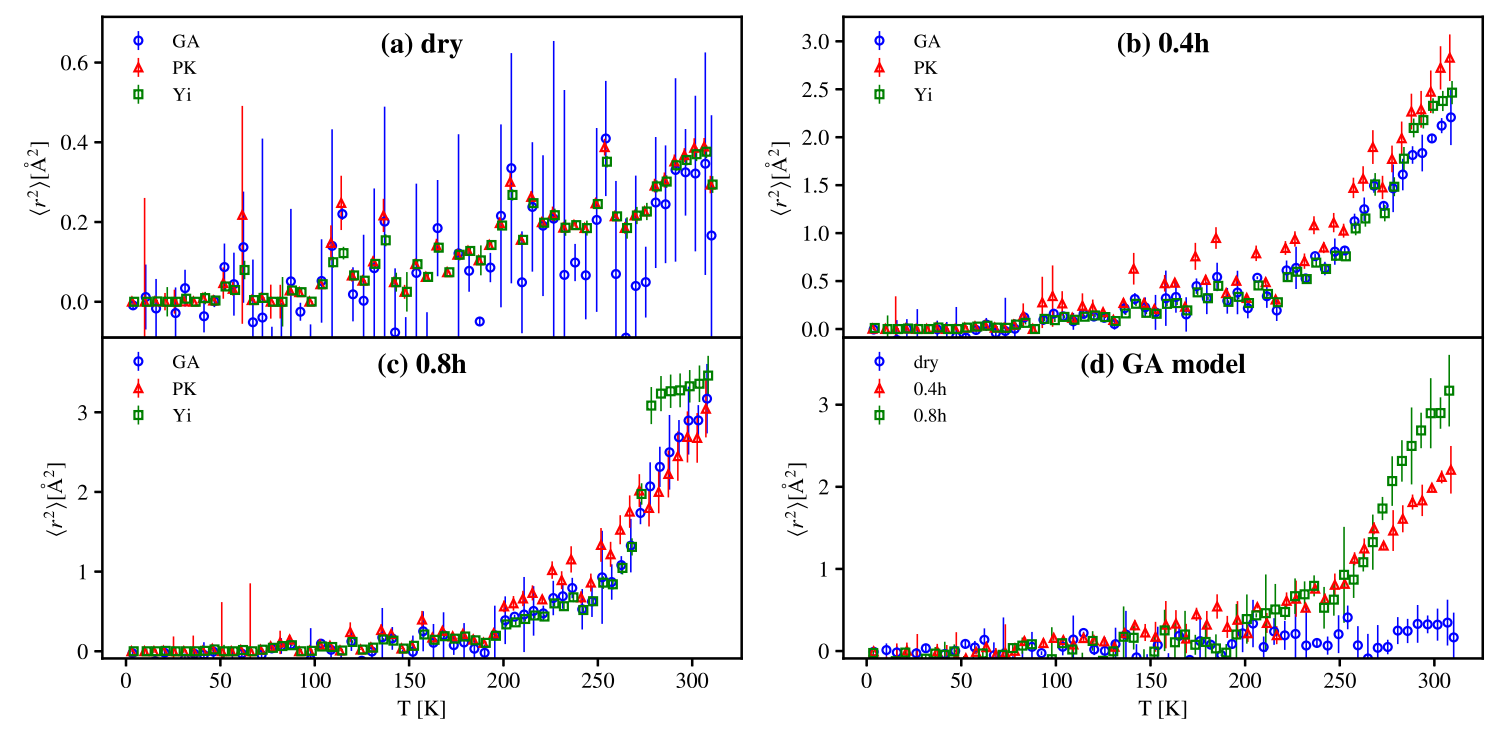

FIG. 9. MSD values extracted from the GA, PK, and Yi models for dry, 0.4 and 0.8 hydrated A-L depleted samples [(a)-(c)]; SPHERES data. (d) shows the MSD of all three hydration levels evaluated with the GA model. For the GA, only the first three Q-values $\left(0.34-0.6 \AA^{-1}\right)$ were used as shown in Fig. 8. 

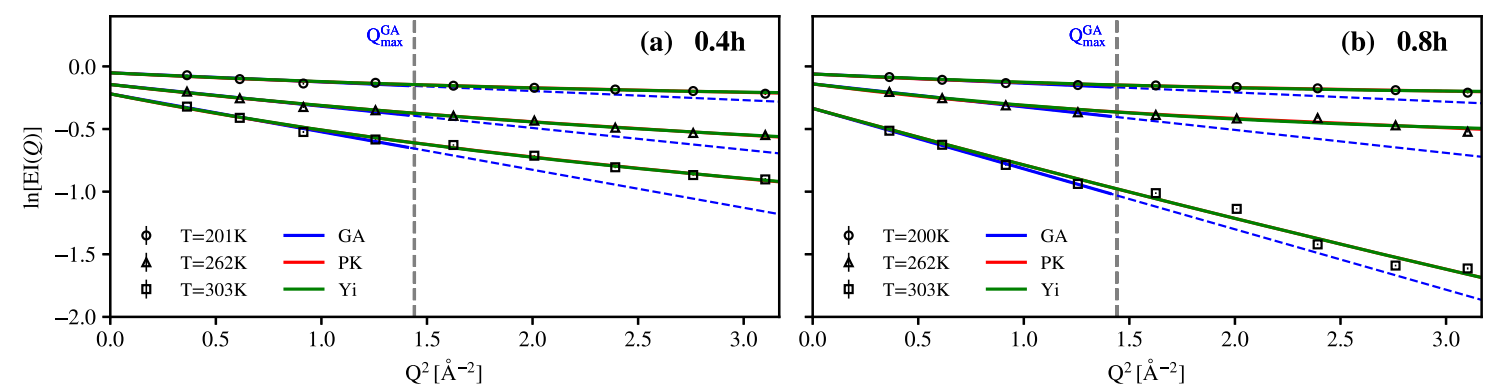

FIG. 10. Representative fits for the three models at three different temperatures for SPHERES data. (a) corresponds to $0.4 \mathrm{~h}$, and (b) corresponds to $0.8 \mathrm{~h}$. The vertical gray line indicates $Q_{\max }=1.2 \AA^{-1}$ used for the GA. Here, the lowest two Q-values are neglected in contrast to Fig. 8.

Figures 11(a)-11(c) summarize the three different hydrations of A-L, as shown before. For all three hydrations, the GA, $\mathrm{PK}$, and Yi models evaluate to similar MSPF/MSD values. The values of the MSD for the dry protein are very similar between the two different Q-ranges of the GA. For the $0.4 \mathrm{~h}$ and $0.8 \mathrm{~h}$ samples, the MSD is lower by a factor of $\approx 2$ compared to fits including the lowest Q-value. Figure 11(d) shows the results of the GA for all three hydrations. A clear difference in the increase of the MSD is visible between the dry and the two hydrated samples at around $220 \mathrm{~K}$. The two hydrated samples are then following the same pattern until around $270 \mathrm{~K}$. At higher temperatures, the $0.8 \mathrm{~h}$ sample has a much larger MSD than the $0.4 \mathrm{~h}$ sample. As explained before, the reason is the melting of frozen $\mathrm{D}_{2} \mathrm{O}$. Nevertheless, it is interesting that both curves have the same MSD until $270 \mathrm{~K}$ which could be due to the non-frozen water shell around the protein being the same in both hydrations.

The comparison between the two different Q-ranges at low Q for the GA [see Figs. 9(a)-9(d) and 11(a)-11(d)] shows that the quantitative value of the MSD is different depending on which range is chosen. The difference of a factor of 2 in the MSD for hydrated samples originates mainly in the difference of the value of the $\operatorname{EI}\left(Q=0 \AA^{-1}\right)$. This is especially visible in the cases of the PK and Yi models. They both use the entire available Q-range at high Q, but fixing $\operatorname{EI}\left(Q=0 \AA^{-1}\right)$ to the respective value obtained by the GA changes their quantitative results dramatically.

\section{High Q-range for GA: 0.96-1.8 $\AA^{-1}$}

As shown in Sec. III C, it is possible to describe only the high $\mathrm{Q}$ range of SPHERES using the GA. The fitted Qrange in which $\ln [\mathrm{EI}(Q)]$ vs $Q^{2}$ is linear is $0.96-1.8 \AA^{-1}$. The resulting MSDs are shown in Fig. 12. Comparing to the low $\mathrm{Q}$ fits without the small angle detectors [Fig. 11(d)], the MSDs are smaller but only up to a factor of 2 in the case of the $0.4 \mathrm{~h}$ sample. More interesting are the changes in the behavior between the different samples. First, it appears that the dry powder shows a slightly larger MSD in the temperature range 150-240 K compared to the hydrated samples which have the same MSD in this temperature range. Such an observation has been reported by Nickels $\mathrm{et} \mathrm{al} .{ }^{52}$ for green fluorescent protein (GFP) [0.4 $\mathrm{D}_{2} \mathrm{O}$ hydration vs. dry, Fig. 2(a) in their publication], which was measured on the very similar instrument, HFBS. They suggest that the frozen hydration shell reduces the MSD at low T by suppressing fast ps fluctuations. A similar behavior was also found in molecular dynamics simulations of GFP by Hong et al. ${ }^{53}$ Second, the MSD of the $0.8 \mathrm{~h}$ sample is the same as that for the dry sample between 240 and $270 \mathrm{~K}$
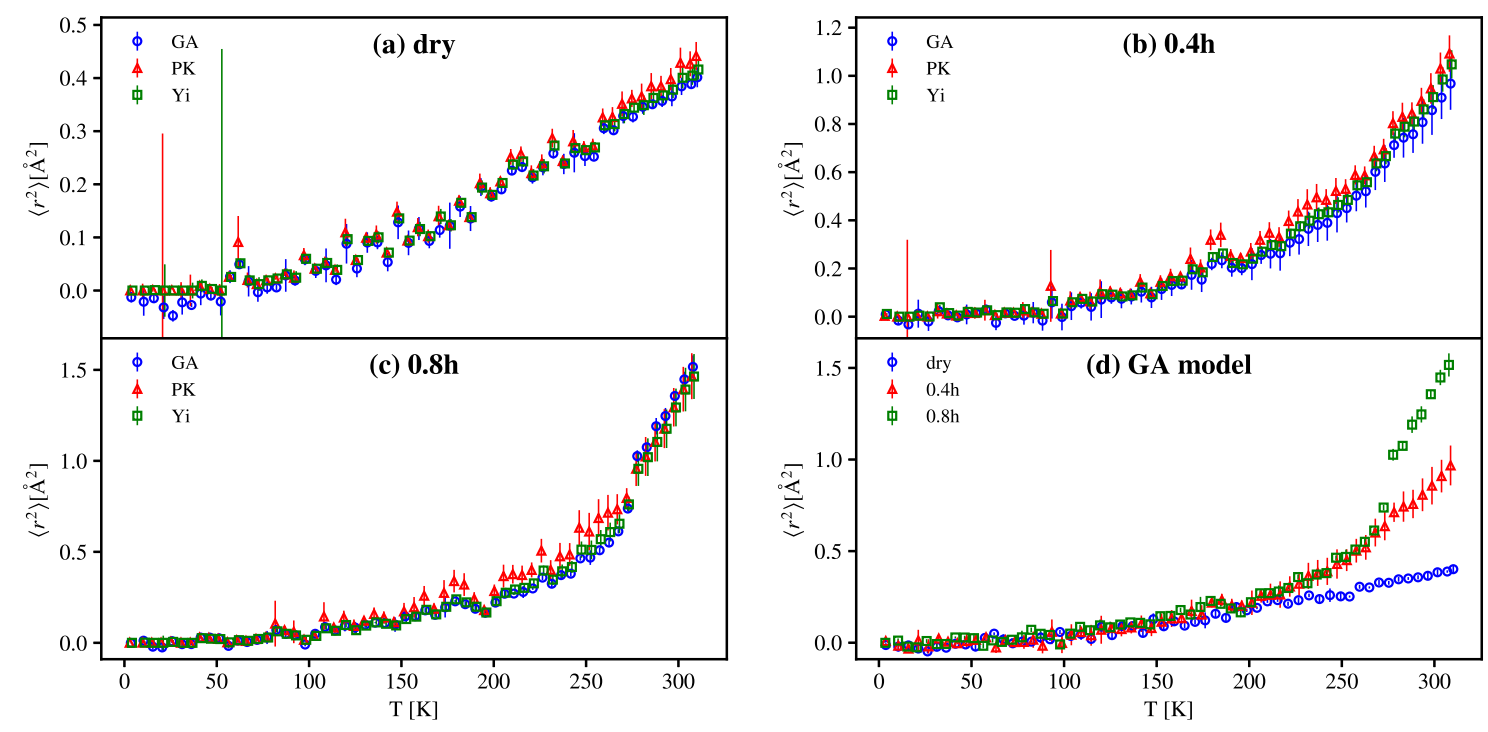

FIG. 11. MSD values extracted from the GA, PK, and Yi models for dry, 0.4, and 0.8 hydrated A-L depleted samples [(a)-(c)]; SPHERES data. (d) shows the MSDs of all three hydration levels evaluated with the GA model. For the GA, the Q-range $0.6-1.2 \AA^{-1}$ is evaluated, and for the PK and Yi models, the Q-range is $0.6-1.8 \AA^{-1}$. 


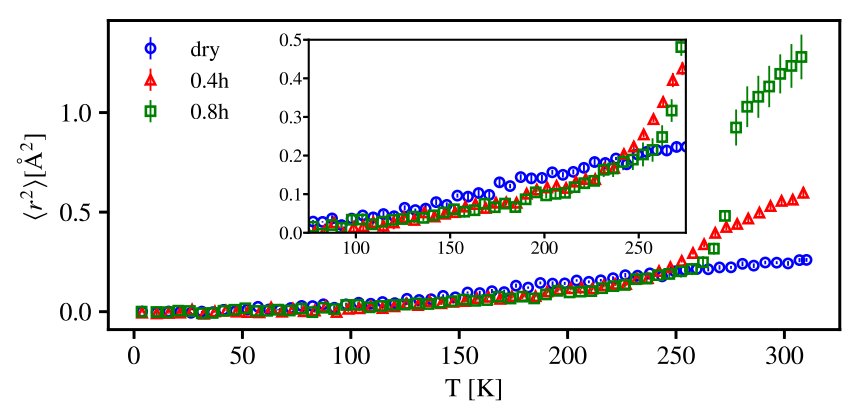

FIG. 12. MSD for all three hydrations evaluated with the GA model at a high $\mathrm{Q}$ range $\left(0.96-1.8 \AA^{-1}\right)$; SPHERES data. The inset shows a zoomed in view of the low $\mathrm{T}$ region.

and lower than for the $0.4 \mathrm{~h}$ sample. It seems that the MSD is inhibited by the frozen $\mathrm{D}_{2} \mathrm{O}$ for the $0.8 \mathrm{~h}$ sample. Above $270 \mathrm{~K}$, the MSD of the $0.8 \mathrm{~h}$ sample increases steeply. This again coincides with the melting point of heavy water at 278 K. Finally, in the inset of Fig. 12, one can see that the dynamical transition still takes place around $200 \mathrm{~K}$, but since the dry sample has a higher MSD at lower T, the crossing between the hydrated and dry sample takes place at $250 \mathrm{~K}$.

\section{The instrument OSIRIS}

For the OSIRIS spectrometer, the chosen Q-range is 0.29$1.5 \AA^{-1}$. Note that OSIRIS has more detectors that cover this
Q-range as compared to the other two spectrometers, allowing for better Q-resolution. The instrumental time resolution is three times larger than on IN13 such that faster motions up to around $25 \mathrm{ps}$ can be probed. This may explain why it is possible to use all three models until $1.5 \AA^{-1}$. Mainly small localized movements are observed and not larger side-chain motions. The resulting MSDs are thus small in comparison to IN13 and SPHERES. Representative fits of the $\operatorname{EI}(Q)$ for the two hydrated samples are shown in Fig. 13.

The MSDs/MSPFs for the different models are illustrated in the same way as for IN13 and SPHERES in Fig. 14. For each hydration, the GA, PK, and Yi models yield almost the same MSD/MSPF values. At $310 \mathrm{~K}$, the PK and Yi models evaluate slightly higher MSPF/MSD values for the dry and 0.4 hydration samples and for the 0.8 hydration sample above $280 \mathrm{~K}$. This behavior confirms that all models give similar results if they use the same Q-range on this instrument. The dry sample shows a linear increase of the MSD/MSPF with increasing temperature. For the 0.4 and 0.8 hydration, the dynamical transition is visible and starts at around $250 \mathrm{~K}$.

\section{Comparison between summed intensities and MSPF}

We would like to note that the MSDs/MSPFs are not always the best way to look at data when small differences are expected between similar samples. In such cases, the
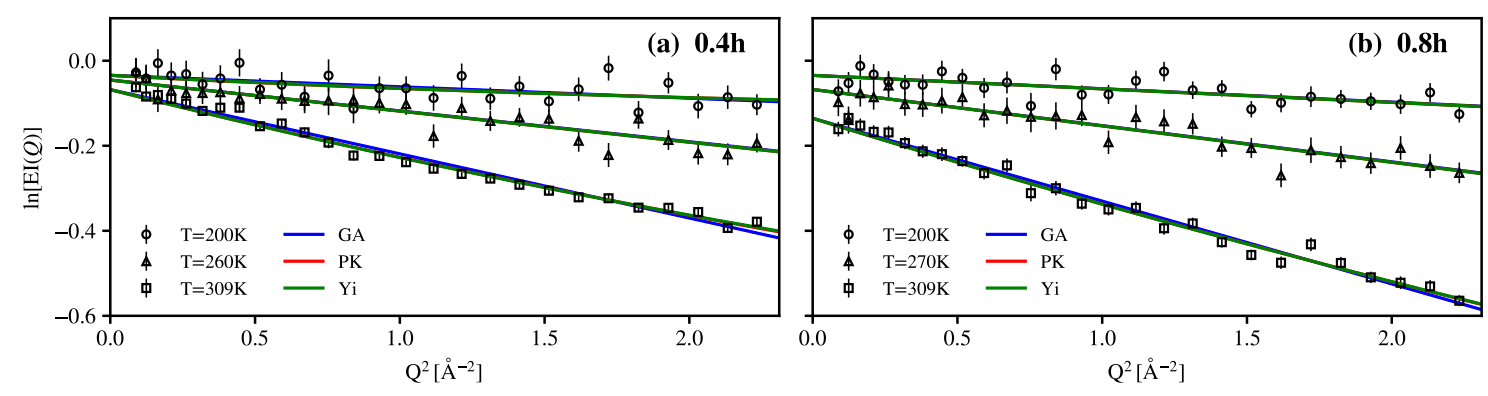

FIG. 13. Representative fits for the three models at three different temperatures for OSIRIS data. (a) corresponds to $0.4 \mathrm{~h}$, and (b) corresponds to $0.8 \mathrm{~h}$.
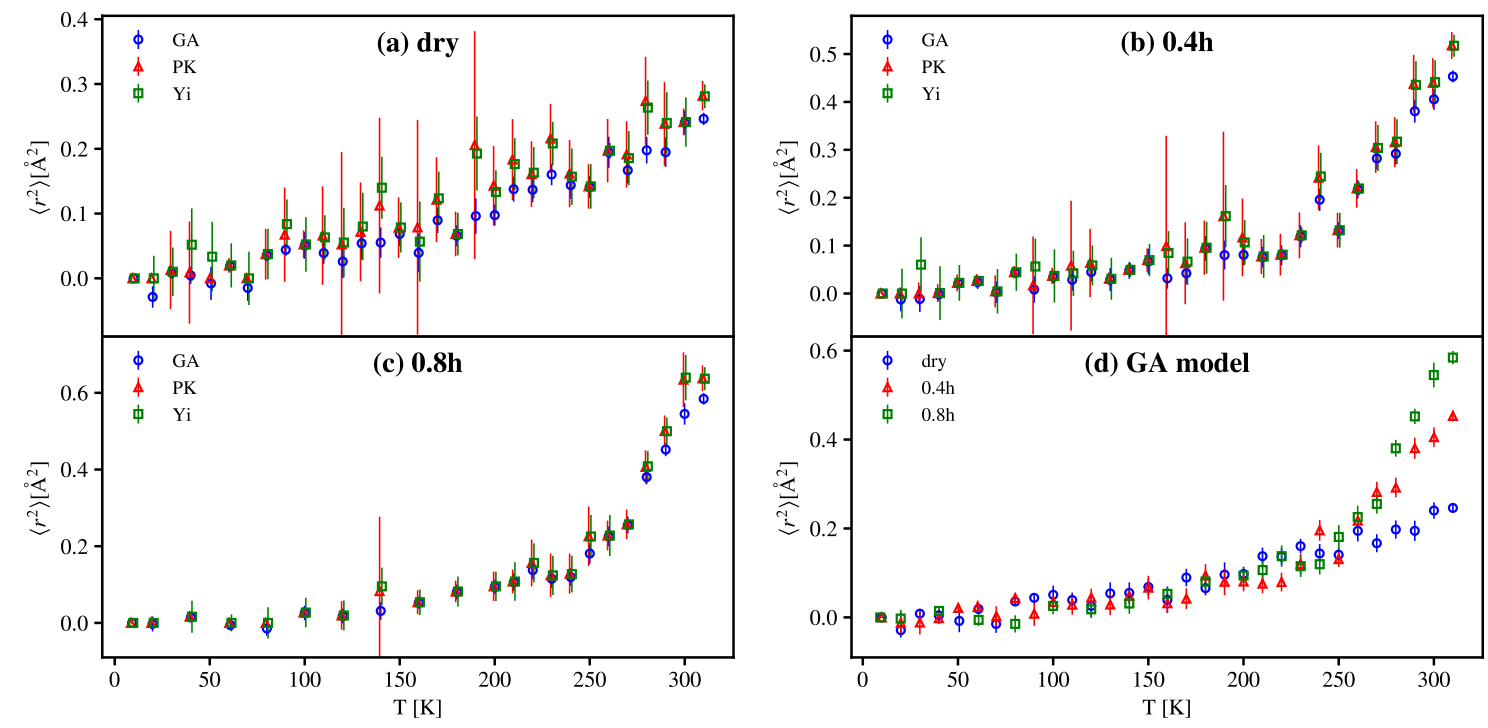

FIG. 14. MSD values extracted from the GA, PK, and Yi models for dry, 0.4, and 0.8 hydrated A-L depleted samples [(a)-(c)]; OSIRIS data. d) shows the MSD of all three hydration levels evaluated with the GA model. All models use the same Q-values $\left(0.29-1.5 \AA^{-1}\right)$. 

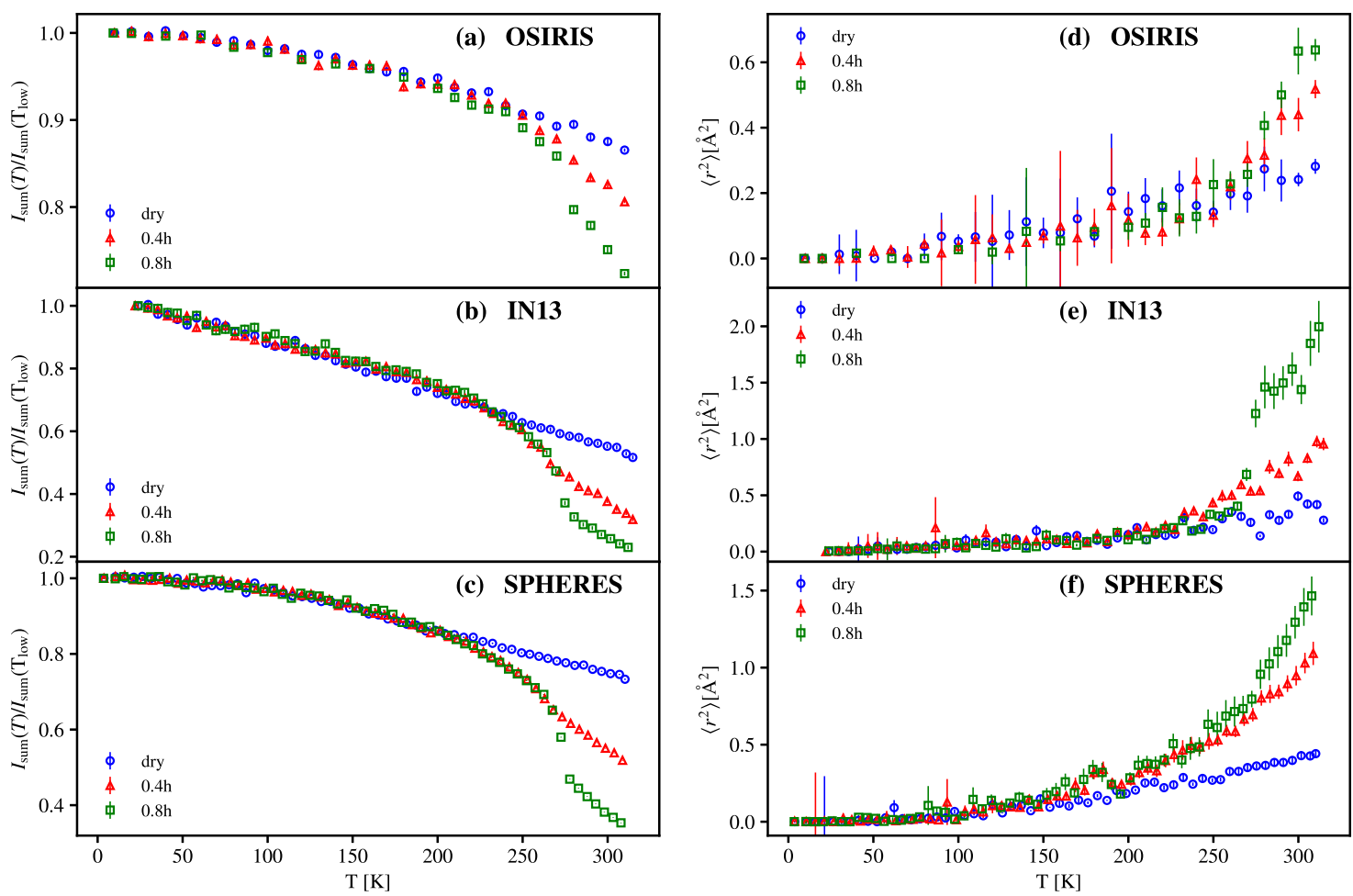

FIG. 15. [(a)-(c)] Summed intensities over the entire available Q-range of each instrument. [(d)-(f)] Respective evaluated MSPF within the PK model over the same Q-range.

comparison of the intensities summed over all (or a range of) available scattering angles, $I_{\text {sum }}$, can be much more insightful. In the limit of the GA, they can moreover be related to the MSD [see Eq. (19)]. We show here the $I_{\text {sum }}$ for the three different instruments and hydration levels in Fig. 15 and compare them to the MSPF evaluated by the PK model using the same Q-range. The results demonstrate the inverse hierarchy for the $I_{\text {sum }}$ and the MSPF between the samples. As the error bars of the summed intensities are much smaller, it allows us to better separate the curves. The differences between the hydration levels become more visible in the summed intensities. For instance, the kink for the $0.8 \mathrm{~h}$ sample at $\approx 275 \mathrm{~K}$ clearly indicates the melting of the ice. Moreover, one distinguishes on IN13 that in the case of $I_{\text {sum }}$ [see Fig. 15(b)], the curve corresponding to the dry sample lies below the curves of the hydrated samples in the temperature domain from 150 to $210 \mathrm{~K}$. We discussed this effect earlier in Sec. IV B 3. This behavior cannot be observed in the MSPF [see Fig. 15(e)] where this information is lost due to data fitting. However, for the MSPF evaluated on SPHERES, a small bump at $180 \mathrm{~K}$ can be seen for the hydrated A-L samples [see Fig. 15(f)], which is not visible in the $I_{\text {sum }}$ [see Fig. 15(c)] and which shows up also when applying the GA [see Fig. 11(d)]. It might be a real effect, eventually due to an ice phase transition, ${ }^{54}$ which appears only through the evaluation of the slopes. Both data analyses might therefore furnish complementary information.

\section{SUMMARY AND CONCLUSION}

We have evaluated a number of models and the resulting MSD/MSPF of A-L for different instruments and hydration levels, and we used various Q-ranges for data analysis. We show that the intercept of $\operatorname{EI}\left(Q=0 \AA^{-1}\right)$ is very important in fitting the data to any given model. Fixing it to the theoretical value of $\operatorname{EI}\left(Q=0 \AA^{-1}\right)=1$ is often not possible for the $\mathrm{GA}$, as shown in Sec. III A, nor for the models that take into account a larger Q-range. Since all models should converge in the limit of $\mathrm{Q} \rightarrow 0$ to the same value, but can present significant variations when leaving it as a free parameter, we advise to fix $\operatorname{EI}(Q=0$ $\AA^{-1}$ ) to a common value for the same dataset. In our case, this value was obtained by the fit of the GA. With such settings, the qualitative results between the models are similar, concerning the dynamical transition and dynamical changes as a function of the hydration level, which leads to the conclusion that the most basic and mainly used model, the GA, is indeed a good standard, if a consistent protocol is followed, i.e., respecting a linear behavior of $\ln [\mathrm{EI}(Q)]$ vs $Q^{2}$ and comparing the same Q-ranges.

The inclusion of the smallest Q-values in traditional reactor-based neutron backscattering spectrometers can change the quantitative MSD dramatically as shown on SPHERES, i.e., resulting in a difference by a factor of two for the MSD. As stated before, the first detectors on SPHERES have a lower resolution than the other detectors, but this is unlikely the only reason for the large differences in MSD. As the temporal range of SPHERES allows us to probe larger amplitudes of motion, it could also arise from the onset of movements of molecular subgroups. Furthermore, if the same Q-range is respected when comparing hydration levels or different samples, the GA is an adequate model and the inclusion of non-Gaussian terms is not needed in order to have a good estimation on the dynamics. By contrast, we show that for a quantitatively accurate analysis, the MSD can be quite 
different between models, especially at a higher hydration level. In fact, even the PK and Yi models do not provide much higher precision, mainly because they are too dependent on the value of $\operatorname{EI}\left(Q=0 \AA^{-1}\right)$. If the experimenter is expecting small differences in dynamics between samples, then the extended models could give more accurate values for the MSD since they include information over a larger Q-range. However, the data will be subject to larger errors than the GA since they also have more free parameters to fit, as can be seen from the MSD/MSPF on OSIRIS (see Sec. IV C). The comparison of the summed intensities $I_{\text {sum }}$ may be more helpful to determine small differences, and they can deviate from the behavior of the MSPF/MSD even when both use the same raw data and Q-ranges (see Sec. IV D).

As EINS is often applied to a class of samples, which have only minor differences (enzymes in the presence or not of an inhibitor, ${ }^{55}$ a wild-type biomolecule against a mutant, ${ }^{56}$ proteins at different concentrations, ${ }^{57}$ with various co-solutes,${ }^{58}$ etc.), the comparison of such a dataset requires highest consistency concerning:

- use of the same hydration level,

- use of the same instrumental resolutions,

- use of the same Q-values for extraction of MSD/MSPF or summed intensities,

- set $\operatorname{EI}\left(Q=0 \AA^{-1}\right)$ to the same value if comparing different models for the same dataset.

In conclusion, our investigation evidences that, despite many efforts to improve the quantitative results for the MSD, significant questions remain and we were not able to establish a reliable method on how to treat the data to get results with highest precision. However, we were able to show that a prevailing although often ignored point is the correct treatment of the point at $\operatorname{EI}\left(Q=0 \AA^{-1}\right)$. Interestingly, our study suggests that in some cases, a bi-modal approach might be sufficient and helpful to distinguish smaller and larger motions as already suggested by, e.g., Nakagawa et al. ${ }^{24}$ Doster and Settles, ${ }^{59}$ or Combet and Zanotti ${ }^{51}$ in the past. On the one hand, such treatment could help to distinguish large motions, which are sometimes associated with movements of hydration water if $\mathrm{H}_{2} \mathrm{O}$ is used or with local translational diffusive displacements, whereas smaller motions account for localized dynamics or vibrations within the biomolecules. Doster very recently also showed that a bi-modal treatment of data taken on myoglobin clearly permits to identify at least two molecular processes which might be sufficient to describe the neutron scattering spectra of proteins. ${ }^{29}$ On the other hand, as discussed in the present work, if a clear separation of linear regimes is no longer appropriate, the models using a continuous distribution of individual MSD describe the dynamics very well without further assumptions. For a reliable conclusion, the exact knowledge of $\operatorname{EI}\left(Q=0 \AA^{-1}\right)$ is of utmost importance, but most instruments do not permit a direct measurement of this value. We suggest further studies by either spin-echo spectroscopy giving access to Q-values as small as $10^{-2} \AA^{-1}$ (IN15 $5^{60}$ at the ILL) or polarisation analysis in combination with neutron techniques (diffraction and/or spectroscopy ${ }^{61,62}$ ) to separate the coherent and incoherent scattering signals. When addressing motions at low Q's, it is important to note that multiple scattering becomes increasingly important and has to be corrected carefully. ${ }^{29}$ This approach would also help us to determine the exact reasons for a deviation from $\operatorname{EI}\left(Q=0 \AA^{-1}\right)=1$ and how it can be estimated. In addition, it may enable more quantitative precision in determining the values of MSD/MSPF in the future.

\section{ACKNOWLEDGMENTS}

D.Z. was supported by a Ph.D. scholarship co-funded by the Communauté Universite Grenoble Alpes, the STFC Rutherford Appleton Laboratory, and the Institut Laue Langevin. J.P. and V.G.S. gratefully acknowledge the support by M. Johnson to partly acquire the Ph.D. grant and fruitful discussions. The authors gratefully acknowledge the financial support provided by JCNS to perform the neutron scattering measurements at the Heinz Maier-Leibnitz Zentrum (MLZ), Garching, Germany. Experiments at the ISIS Neutron and Muon Source were supported by a beamtime allocation from the Science and Technology Facilities Council. We thank the ILL for beamtime allocation.

${ }^{1}$ B. P. Schoenborn, Nature 224, 143 (1969).

${ }^{2}$ W. Doster, S. Cusack, and W. Petry, Nature 337, 754 (1989).

${ }^{3}$ L. Rusevich, V. García Sakai, B. Franzetti, M. Johnson, F. Natali, E. Pellegrini, J. Peters, J. Pieper, M. Weik, and G. Zaccai, Eur. Phys. J. E 36, 80 (2013).

${ }^{4}$ M. Bée, Quasielastic Neutron Scattering: Principles and Applications in Solid State Chemistry, Biology and Materials Science (Adam Hilger, Philadelphia, 1988).

${ }^{5}$ L. Van Hove, Phys. Rev. 95, 249 (1954).

${ }^{6}$ H. Frauenfelder, R. D. Young, and P. W. Fenimore, Proc. Natl. Acad. Sci. U. S. A. 114, 5130 (2017), http://www.pnas.org/content/114/20/5130.full.pdf.

${ }^{7}$ G. R. Kneller, Proc. Natl. Acad. Sci. U. S. A. 115, 9450 (2018), http://www.pnas.org/content/early/2018/08/29/1718720115.full.pdf.

${ }^{8}$ W. Doster, H. Nakagawa, and M. Appavou, J. Chem. Phys. 139, 045105 (2013).

${ }^{9}$ F. Gabel, B. Bicout, U. Lehnert, M. Tehei, M. Weik, and G. Zaccai, Q. Rev. Biophys. 35, 327 (2002).

${ }^{10}$ G. Zaccai, J. Non-Cryst. Solids 357, 615 (2011), 6th International Discussion Meeting on Relaxation in Complex Systems.

${ }^{11} \mathrm{G}$. Zaccai, Science 288, 1604 (2000).

${ }^{12}$ M. Trapp, J. Marion, M. Tehei, B. Deme, T. Gutberlet, and J. Peters, Phys. Chem. Chem. Phys. 15, 20951 (2013).

${ }^{13}$ J. Roh, V. Novikov, R. Gregory, J. Curtis, Z. Chowdhuri, and A. Sokolov, Phys. Rev. Lett. 95, 038101 (2005).

${ }^{14} \mathrm{G}$. Squires, Introduction to the Theory of Thermal Neutron Scatering (Dover Publications, 1978).

${ }^{15}$ M. T. Telling, S. Howells, J. Combet, L. A. Clifton, and V. García Sakai, Chem. Phys. 424, 32 (2013), Neutron Scattering Highlights on Water and Biological Systems.

${ }^{16}$ W. Doster, M. Diehl, W. Petry, and M. Ferrand, Physica B 301, 65 (2001).

${ }^{17}$ A. Rahman, K. S. Singwi, and A. Sjolander, Phys. Rev. 126, 986 (1962).

${ }^{18}$ G. R. Kneller and G. Chevrot, J. Chem. Phys. 137, 225101 (2012).

${ }^{19}$ A. Tokuhisa, Y. Joti, H. Nakagawa, A. Kitao, and M. Kataoka, Phys. Rev. E 75, 041912 (2007).

${ }^{20}$ T. Becker and J. C. Smith, Phys. Rev. E 67, 021904 (2003).

${ }^{21}$ Z. Yi, Y. Miao, J. Baudry, N. Jain, and J. C. Smith, J. Phys. Chem. B 116, 5028 (2012).

${ }^{22}$ A. Rahman, Phys. Rev. 136, A405 (1964).

${ }^{23}$ K. Sköld, J. M. Rowe, G. Ostrowski, and P. D. Randolph, Phys. Rev. A 6, 1107 (1972).

${ }^{24}$ H. Nakagawa, H. Kamikubo, I. Tsukushi, T. Kanaya, and M. Kataoka, J. Phys. Soc. Jpn. 73, 491 (2004).

${ }^{25}$ L. Meinhold, D. Clement, M. Tehei, R. Daniel, J. L. Finney, and J. C. Smith, Biophys. J. 94, 4812 (2008).

${ }^{26}$ J. Peters and G. R. Kneller, J. Chem. Phys. 139, 165102 (2013).

${ }^{27}$ G. R. Kneller and K. Hinsen, J. Chem. Phys. 131, 045104 (2009). 
${ }^{28}$ D. Vural, J. C. Smith, and H. R. Glyde, Biophys. J. 114, 2397 (2018).

${ }^{29}$ W. Doster, Int. J. Mol. Theor. Phys. 2, 1 (2018).

${ }^{30}$ D. Vural, L. Hong, J. C. Smith, and H. R. Glyde, Phys. Rev. E 91, 052705 (2015).

${ }^{31}$ S. Perticaroli, G. Ehlers, C. B. Stanley, E. Mamontov, H. O'Neill, Q. Zhang, X. Cheng, D. A. Myles, J. Katsaras, and J. D. Nickels, J. Am. Chem. Soc. 139, 1098 (2017).

${ }^{32}$ V. F. Sears, Neutron News 3, 26 (1992).

${ }^{33}$ F. Natali, J. Peters, D. Russo, S. Barbieri, C. Chiapponi, A. Cupane, A. Deriu, M. T. Di Bari, E. Farhi, Y. Gerelli, P. Mariani, A. Paciaroni, C. Rivasseau, G. Schiró, and F. Sonvico, Neutron News 19, 14 (2008).

${ }^{34} \mathrm{D}$. Zeller and J. Peters, Internal time on IN13 (dataset), Inst. Laue-Langevin (ILL) (2016).

${ }^{35}$ J. Wuttke, A. Budwig, M. Drochner, H. Kammerling, F. J. Kayser, H. Kleines, V. Ossovyi, L. C. Pardo, M. Prager, D. Richter, G. J. Schneider, H. Schneider, and S. Staringer, Rev. Sci. Instrum. 83, 075109 (2012).

${ }^{36}$ Heinz Maier-Leibnitz Zentrum, J. Large-Scale Res. Facil. 1, A30 (2015).

${ }^{37}$ M. T. F. Telling and K. H. Andersen, Phys. Chem. Chem. Phys. 7, 1255 (2005).

${ }^{38}$ D. Richard, M. Ferrand, and G. J. Kearley, J. Neutron Res. 4, 33 (1996).

${ }^{39}$ O. Arnold, J. Bilheux, J. Borreguero, A. Buts, S. Campbell, L. Chapon, M. Doucet, N. Draper, R. F. Leal, M. Gigg, V. Lynch, A. Markvardsen, D. Mikkelson, R. Mikkelson, R. Miller, K. Palmen, P. Parker, G. Passos, T. Perring, P. Peterson, S. Ren, M. Reuter, A. Savici, J. Taylor, R. Taylor, R. Tolchenov, W. Zhou, and J. Zikovsky, Nucl. Instrum. Methods Phys. Res., Sect. A 764, 156 (2014).

${ }^{40}$ M. Jasnin, L. van Eijck, M. M. Koza, J. Peters, C. Laguri, H. Lortat-Jacob, and G. Zaccai, Phys. Chem. Chem. Phys. 12, 3360 (2010).

${ }^{41}$ M. Trapp, M. Trovaslet, F. Nachon, M. M. Koza, L. van Eijck, F. Hill, M. Weik, P. Masson, M. Tehei, and J. Peters, J. Phys. Chem. B 116, 14744 (2012).

${ }^{42}$ V. Réat, G. Zaccai, C. Ferrand, and C. Pfister, Biological macromolecular dynamcis, Proceedings of a Workshop on Inelastic and Quasielastic Neutron Scattering in Biology (Adenine Press, 1996), pp. 117-122.

${ }^{43}$ Z. Liu, J. Huang, M. Tyagi, H. O’Neill, Q. Zhang, E. Mamontov, N. Jain, Y. Wang, J. Zhang, J. C. Smith, and L. Hong, Phys. Rev. Lett. 119, 048101 (2017).

${ }^{44}$ DLMF: NIST Digital Library of Mathematical Functions, http://dlmf.nist.gov/, Release 1.0 .18 of 2018-03-27, edited by f. W. J.
Olver, A. B. Olde Daalhuis, D. W. Lozier, B. I. Schneider, R. F. Boisvert, C. W. Clark, B. R. Miller, and B. V. Saunders (NIST, 2018).

${ }^{45}$ M. Newville, T. Stensitzki, D. B. Allen, and A. Ingargiola, LMFIT: NonLinear Least-Square Minimization and Curve-Fitting for Python (2014), available at https://doi.org/10.5281/zenodo.11813.

${ }^{46}$ M. Tehei, R. Daniel, and G. Zaccai, Eur. Biophys. J. 35, 551 (2006).

${ }^{47}$ J. Wuttke, Phys. Rev. E 62, 6531 (2000).

${ }^{48}$ J. Wuttke and M. Zamponi, Rev. Sci. Instrum. 84, 115108 (2013).

${ }^{49}$ U. Lehnert, V. Reat, M. Weik, G. Zaccai, and C. Pfister, Biophys. J. 75, 1945 (1998).

${ }^{50}$ J. M. Zanotti, M. C. Bellissent Funel, and S. Chen, Europhys. Lett. 71, 91 (2005).

${ }^{51}$ S. Combet and J. M. Zanotti, Phys. Chem. Chem. Phys. 14, 4927 (2012).

${ }^{52}$ J. D. Nickels, H. O'Neill, L. Hong, M. Tyagi, G. Ehlers, K. L. Weiss, Q. Zhang, Z. Yi, E. Mamontov, J. C. Smith, and A. P. Sokolov, Biophys. J. 103, 1566 (2012).

${ }^{53}$ L. Hong, D. C. Glass, J. D. Nickels, S. Perticaroli, Z. Yi, M. Tyagi, H. O'Neill, Q. Zhang, A. P. Sokolov, and J. C. Smith, Phys. Rev. Lett. 110, 028104 (2013).

${ }^{54}$ C. D. Andersson, N. Martinez, D. Zeller, A. Allgardsson, M. M. Koza, B. Frick, F. Ekstrüm, J. Peters, and A. Linusson, J. Phys. Chem. B 122, 8516 (2018).

${ }^{55}$ J. Peters, N. Martinez, M. Trovaslet, K. Scannapieco, M. M. Koza, P. Masson, and F. Nachon, Phys. Chem. Chem. Phys. 18, 12992 (2016).

${ }^{56}$ S. Sacquin-Mora, P. Sebban, V. Derrien, B. Frick, R. Lavery, and C. AlbaSimionesco, Biochemistry 46, 14960 (2007).

${ }^{57}$ M. Erlkamp, J. Marion, N. Martinez, C. Czeslik, J. Peters, and R. Winter, J. Phys. Chem. B 119, 4842 (2015).

${ }^{58}$ A. Paciaroni, E. Cornicchi, A. De Francesco, M. Marconi, and G. Onori, Eur. Biophys. J. 35, 591 (2006).

${ }^{59}$ W. Doster and M. Settles, Biochim. Biophys. Acta, Proteins Proteomics 1749, 173 (2005).

${ }^{60}$ See https://www.ill.eu/fr/users-en/instruments/instruments-list/in15/ characteristics/ for information about the IN15 instrument characteristics; accessed 25 July 2018.

${ }^{61}$ A. M. Gaspar, S. Busch, M.-S. Appavou, W. Haeussler, R. Georgii, Y. Su, and W. Doster, Biochim. Biophys. Acta, Proteins Proteomics 1804, 76 (2010), Includes Special Section: Protein-Water Interactions.

${ }^{62}$ G. M. Paternò, J. R. Stewart, A. Wildes, F. Cacialli, and V. García Sakai, Polymer 105, 407 (2016) . 